\title{
Analysis of the performance of closely spaced low-cost multi-GNSS receivers
}

\author{
Chenyu Xue $^{1}$ (D) Panos Psimoulis ${ }^{1} \cdot$ Qiuzhao Zhang $^{2} \cdot$ Xiaolin Meng $^{1}$
}

Received: 24 April 2020 / Accepted: 13 January 2021 / Published online: 26 January 2021

(C) The Author(s) 2021

\begin{abstract}
The recent advances of low-cost GNSS receivers have broadened their application field not only in positioning and navigation, but also in deformation monitoring of civil engineering structures and geohazards. Even though some consumer-grade low-cost GNSS receivers can achieve cm-level accuracy, their lower performance compared to the dual-frequency high-end GNSS receivers restricts its systematic application of GNSS technology in monitoring projects. In this study, the noise level and performance of the low-cost GNSS receivers are assessed against geodetic receivers in terms of precision and availability when subjected to different measurements conditions, such as antenna grade, satellite constellation, and base station (antenna-receiver), based on zero- and short-baseline measurements. Furthermore, a new method is developed where a dual low-cost GNSS rover-system is formed by deploying two closely spaced low-cost GNSS receivers (30 cm apart), aiming to model their common error (multipath, satellite constellation, etc.) and reduce their noise level. The analysis of the zero- and short-baseline measurements reveals the potential improvement of the precision of the low-cost receiver by using multi-GNSS measurements and the importance of using a GNSS base station with geodetic antenna. However, development of a methodology which is based on adopting the sidereal filtering and the common mode error technique for the two closely spaced low-cost GNSS receivers may lead to precision of mmlevel. The proposed methodology may broaden the application of low-cost GNSS receivers in monitoring networks and mainly for slowly developed deformations.
\end{abstract}

Keywords Multi-GNSS $\cdot$ Low-cost GNSS receivers $\cdot$ Zero baseline $\cdot$ Short baseline $\cdot$ Multipath $\cdot$ Common mode error

\section{Introduction}

The GNSS technology has been established in the last decades for deformation monitoring applications, as the development of the GNSS receivers and processing methods led towards a more precise and accurate positioning, especially in shortbaseline (SBL) applications (Häberling et al. 2015; Moschas

Chenyu Xue

isxcx@nottingham.ac.uk

1 Nottingham Geospatial Institute, The University of Nottingham, Triumph Rd, Nottingham NG7 2TU, UK

2 School of Environmental Science and Spatial Informatics, China University of Mining and Technology, Xuzhou, Jiangsu Province, China and Stiros 2013; Psimoulis et al. 2008; Psimoulis and Stiros 2008). However, the requirement of dual frequency receiver to achieve accuracy of a few mm- to cm-level leads to significantly high cost of a GNSS monitoring station and consequently limits its applications in deformation monitoring (Caldera et al. 2016). On the other hand, the potential of multi-GNSS measurement was made feasible through development of other satellite positioning systems (Galileo, BeiDou, etc.; Roberts et al. 2018), and the advances in lowcost receivers (e.g., $10 \mathrm{~Hz}$ sampling-rate; Wilkinson et al. 2017), broaden their potential in navigation (GarridoCarretero et al. 2019; Willi and Rothacher 2017); agricultural (Takahashi et al. 2015) and monitoring applications (Biagi et al. 2016; Krietemeyer et al. 2018).

The recently developed commercial single-frequency lowcost GNSS receivers have improved performance achieving $\mathrm{cm}$-level or even higher accuracy for static positioning (Cina 
and Piras 2015; Takasu and Yasuda 2009), and it is found that under favorable circumstances, such as long acquisition time, short baseline length, and use of external antenna, the difference between consumer-grade (u-blox 5T, etc) and geodetic grade receivers is not large (Cina and Piras 2015). However, most single-frequency low-cost GNSS station (low-cost receiver with patch antenna) still cannot have the robustness and reliability of the high-end GNSS stations (dual frequency GNSS receiver and geodetic antenna). The difference in the design/configuration between the two types of the receivers/antennas results to the high noise level, low multipath resistance, and poor ambiguity resolution of the low-cost receiver relatively to the high-end GNSS stations (Takasu and Yasuda 2009).

Recent studies have focused on the evaluation of the performance of the low-cost GNSS receivers in deformation monitoring (Caldera et al. 2016; Jo et al. 2013; Zhang and Schwieger 2016). Jo et al. (2013) assessed the accuracy of low-cost GPS receivers in the monitoring of a few dm-level displacements, indicating that the accuracy of low-cost GNSS system is adequate for deformation of 20-30 cm level of dynamic displacement. Caldera et al. (2016) analyzed the short baseline result of a low-cost monitoring system using different software, showing that a few mm level of movement could be detected with the daily short baseline solutions. Zhang and Schwieger (2016) analyzed the temporal and spatial correlation of an array of GPS stations consisted of low-cost receivers.

The main hypothesis of this study is that the combination of two closely spaced low-cost GNSS receivers (up to a few dm in-between distance) allows to model the common errors between the two receivers, due to almost identical surrounding environment of the two GNSS receivers, which can be used to reduce their common measurements noise. Based on that hypothesis, we develop the dual low-cost GNSS system, a formation of two closely spaced $(\sim 30 \mathrm{~cm})$ low-cost GNSS receivers, and we examined how the precision of the GNSS coordinate time-series can be enhanced by applying three different approaches or combination of those. The three approaches are as follows: (i) the application of multi-GNSS satellite constellation (GPS, GLONASS, Galileo) to enhance the GNSS solution precision, (ii) the application of the sidereal filter (SDF) to model the multipath error of each GNSS receiver and limit it in the GNSS coordinate time-series, and (iii) the application of the common mode error (CME) of the two closely space low-cost GNSS receivers, in order to model the common errors between the two receivers and subtracted it from their GNSS coordinate time-series.

The multi-GNSS solution is an approach broadly used to enhance the precision of the GPS solution, based on the contribution of the recently developed satellite systems, such as BeiDou and Galileo. The SDF is a commonly applied method, mainly for GPS measurements, which is based on the repetition of the satellite constellation every sidereal day. Hence, for a permanent GPS station, the same measuring environment is created by the repetition of GPS satellite orbits and the constellation related errors, such as multipath, can be identified and mitigated by applying the SDF (Ragheb et al. 2007; Zhong et al. 2010; Peppa et al. 2018). The CME method, which was developed by Wdowinski et al. (1997), states that the common mode signal could be mitigated by a filtering algorithm (stacking) if a network of GPS stations are creating sufficiently short baselines $(50 \mathrm{~m})$. This method assumes that the GPS stations are subjected practically to similar ionospheric, tropospheric, and multipath conditions, which allows to model the common pattern of the measurements noise using all the GPS receivers and subtract this modelled noise from each GPS time series. The CME is broadly applied in GNSS
Fig. 1 Deployment of the zerobaseline GNSS measurements (left) with the Leica AS10 antenna (20/03/2018) and (right) with patch antenna $(22 / 03 / 2018)$
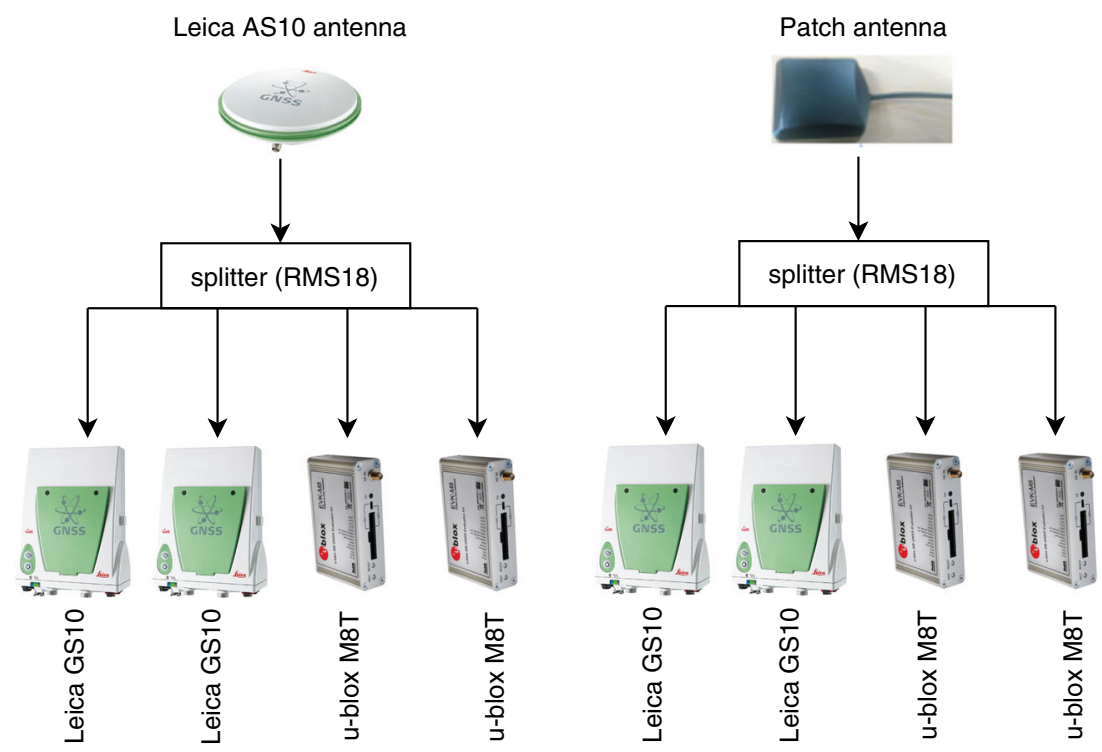
Table 1 Different combinations of solutions for the zero-baseline GNSS measurements

\begin{tabular}{llll}
\hline Combination & Base receiver & Rover receiver & Antenna \\
\hline A & u-blox M8T & u-blox M8T & patch antenna \\
B & GS10 & GS10 & patch antenna \\
C & GS10 & u-blox M8T & patch antenna \\
D & u-blox M8T & u-blox M8T & AS10 \\
E & GS10 & GS10 & AS10 \\
F & GS10 & u-blox M8T & AS10 \\
\hline
\end{tabular}

permanent networks in order to remove the common errors between the GNSS stations (Williams et al. 2004).

The novelty of this study is that we developed a method where two low-cost GNSS receivers are combined in closely spaced formation and by applying combination of different approaches (multi-GNSS, SDF, CME), the precision of lowcost GNSS receivers is enhanced significantly reaching even up to 1-2 mm-level, making more applicable for shortbaseline deformation monitoring applications. Even though, previous studies have focused on low-cost GNSS receivers precision (Caldera et al. 2016; Jo et al. 2013; Zhang and Schwieger 2016), in none of those this closely spaced multiGNSS formation and the different approaches were applied in order to enhance the performance of the low-cost GNSS receivers. To develop this method, we need to understand the performance of low-cost receivers by following a holistic approach in assessing the impact of several parameters (satellite systems, antenna type, quality of base station) on the precision of the low-cost GNSS receiver, with respect the precision of dual-frequency GNSS receiver, based on zero- and shortbaseline GNSS measurements.

\section{Zero- and short-baseline GNSS measurements}

Two major approaches for the evaluation of the GNSS receiver noise are the zero-baseline (ZBL) and short-baseline (SBL) measurements (Van Sickle 2001). The ZBL measurement is a well-known method to determine the receiver noise (Tang et al. 2018; Roberts et al. 2018), while the short-baseline measurements are used to evaluate the impact of other error sources (e.g., multipath, antenna, etc.) of the GNSS measurements (Moschas and Stiros 2011).

In ZBL measurements, two or more GNSS receivers are connected with the same antenna, where the double-difference (DD) processing using one receiver as base and the other(s) as rover(s) leads to the cancellation of errors, such as due to orbit/ clock errors, atmospheric delays (ionosphere, troposphere), antenna (Gourevitch 1996), and multipath (Amiri-Simkooei and Tiberius 2007). The main remaining error is due to the geometry of the satellite constellation and the receiver noise.

On the other hand, in SBL measurements, errors due to atmosphere delays, clock errors, etc can be significantly limited due to the relatively short length (Raquet 1996). However, multipath errors cannot be limited due to the site-specific multipath environment of the base and rover receiver. The SBL experimental GNSS measurements represent the conditions which are met in deformation monitoring applications, where the baseline are of relatively short length (up to a few $\mathrm{km}$ ) and main error source the multipath effect (Meng et al. 2018).
Fig. 2 E, N, and U coordinate time-series of combination D zero-baseline measurements for the four available solutions: G:GPS-only, G+R:GPS+ GLONASS, G+R+E:GPS+ GLONASS+Galileo, and G+ E:GPS+Galileo. The G+E solution seems to be the most precise (i.e., the least variance), while $\mathrm{G}+\mathrm{R}$ solution seems to have the most outliers
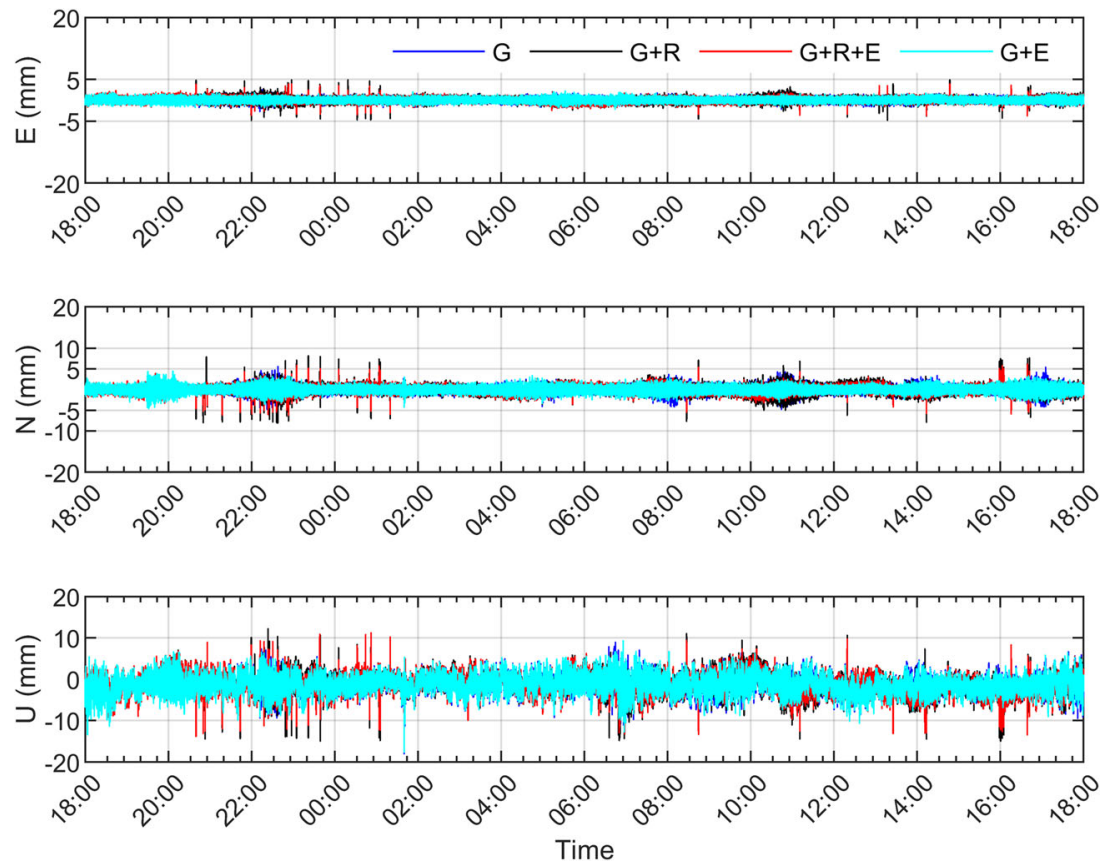
Fig. 3 E, N, and U coordinate time-series of combination $\mathrm{A}$ zero-baseline measurements for the four available solutions: $\mathrm{G}$ : GPS-only, G+R:

GPS+GLONASS, G+R+E: GPS+GLONASS+Galileo, and G+E: GPS+Galileo. The performance of the different multi-GNSS solutions is similar to those of combination D
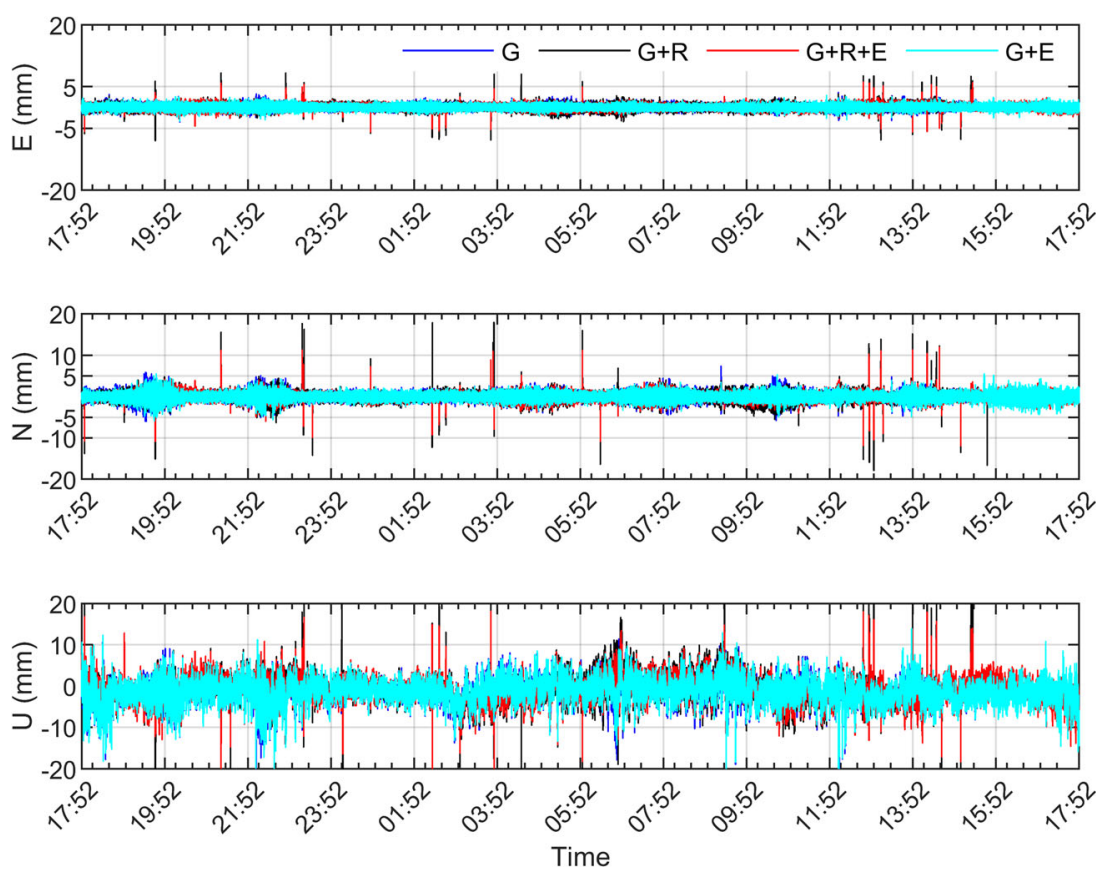

\section{Zero-baseline GNSS experiment}

\section{Zero-baseline GNSS measurements}

The ZBL experiment took place on the roof of Nottingham Geospatial Building (NGI) from 20/03/2018 to 24/03/2018, at the University of Nottingham, UK, where four GNSS receivers were connected to one antenna (geodetic or patch antenna). The aim was to evaluate the performance of a low-cost GNSS receiver against dual-frequency GNSS receiver, for patch and geodetic antenna and for available satellite constellations of different satellite systems. Two low-cost single frequency (L1) u-blox (M8T module) GNSS receivers were used, with the capacity to record multi-constellation carrier phase measurements, with nominal temporal accuracy of sub-microsecond (Wilkinson et al. 2017). For dual frequency GNSS receivers, two Leica GS10 GNSS receivers were used with nominal horizontal precision of $8 \mathrm{~mm} \pm 1 \mathrm{ppm}$ and vertical

Table 2 Mean average and standard deviation of combination A zerobaseline measurements for the four solutions (GPS: G; GPS+GLONASS: G+R; GPS+GLONASS+Galileo: G+R+E; GPS+Galileo: G+E), which are presented in Fig. 2

\begin{tabular}{|c|c|c|c|c|c|c|}
\hline \multirow[b]{2}{*}{ Unit (mm) } & \multicolumn{2}{|c|}{ E component } & \multicolumn{2}{|c|}{$\mathrm{N}$ component } & \multicolumn{2}{|c|}{ U component } \\
\hline & mean & $\sigma$ & mean & $\sigma$ & mean & $\sigma$ \\
\hline G & 0.01 & 0.47 & 0.00 & 0.77 & -1.33 & 1.99 \\
\hline $\mathrm{G}+\mathrm{R}$ & 0.03 & 0.54 & -0.02 & 0.85 & -1.32 & 2.14 \\
\hline $\mathrm{G}+\mathrm{R}+\mathrm{E}$ & 0.01 & 0.48 & -0.00 & 0.72 & -1.32 & 2.06 \\
\hline $\mathrm{G}+\mathrm{E}$ & 0.01 & 0.44 & 0.02 & 0.69 & -1.36 & 2.01 \\
\hline
\end{tabular}

precision of $15 \mathrm{~mm} \pm 1 \mathrm{ppm}$ for post-processing in kinematic mode (Leica Geosystems 2012). Two different types of antennas were used both with the capacity to receive multiconstellation GNSS signals: (i) a low-cost patch antenna from the EVK-M8T kit with higher gain on large ground plane and (ii) a geodetic antenna (Leica AS10) commonly used in monitoring applications. Figure 1 shows the deployment of the zero-baseline measurements, where on 20/03/2018, the GNSS receivers were connected through signal splitter (RMS18 Rack Mount Splitter) with the Leica AS10 and on 22/03/2018 with the patch antenna respectively, tracking the signal of GPS, GLONASS and Galileo satellites. The RMS18 signal splitter is manufactured by GPS source, with $12 \mathrm{~dB}$ typical gain and capacity to pass GPS, GLONASS and Galileo signal, and it was used as a port of signal transmission. The sampling-rate of the measurements for both days was 1 Hz. A computer for logging the low-cost receivers observations was used.

Table 3 Mean average and standard deviation of combination D zerobaseline measurements for the four solutions (GPS: G; GPS+GLONASS: G+R; GPS+GLONASS+Galileo: G+R+E; GPS+Galileo: G+E), which are presented in Fig. 3

\begin{tabular}{|c|c|c|c|c|c|c|}
\hline \multirow[b]{2}{*}{ Unit (mm) } & \multicolumn{2}{|c|}{ E component } & \multicolumn{2}{|c|}{$\mathrm{N}$ component } & \multicolumn{2}{|c|}{ U component } \\
\hline & mean & $\sigma$ & mean & $\sigma$ & mean & $\sigma$ \\
\hline G & 0.02 & 0.55 & 0.00 & 0.89 & -1.40 & 2.79 \\
\hline $\mathrm{G}+\mathrm{R}$ & 0.03 & 0.62 & -0.05 & 0.95 & -1.04 & 2.86 \\
\hline $\mathrm{G}+\mathrm{R}+\mathrm{E}$ & 0.02 & 0.55 & -0.02 & 0.81 & -1.08 & 2.73 \\
\hline $\mathrm{G}+\mathrm{E}$ & 0.02 & 0.52 & 0.01 & 0.82 & -1.41 & 2.78 \\
\hline
\end{tabular}


Fig. 4 Spectra of $E, N$, and $U$ components for the solutions of the combinations $\mathrm{A}, \mathrm{D}$, and $\mathrm{E}$ using GPS-only constellation. It is evident that combination $\mathrm{E}$ (Leica receiver and antenna) result to the least noisy spectrum. Also, the geodetic antenna reduces the u-blox receiver noise, with respect the combination of patch antenna, mainly in the Up component
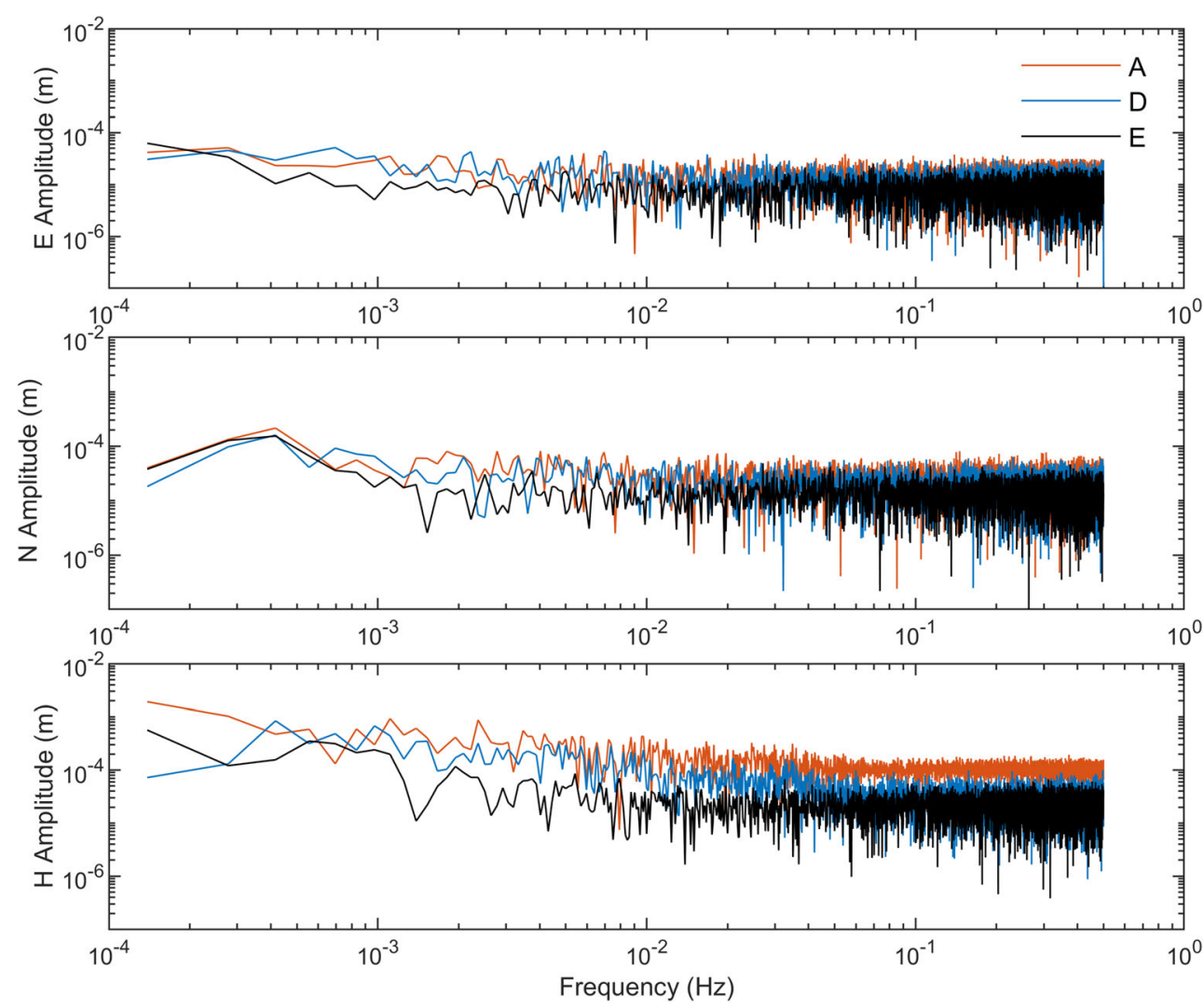

\section{GNSS data process and methodology of time-series analysis}

The GNSS records of u-blox and GS10 receivers were converted to Rinex files, using Teqc software, which then were processed in RTK-Lib 2.4.3 (Takasu and Yasuda 2009), software commonly used on GNSS monitoring applications
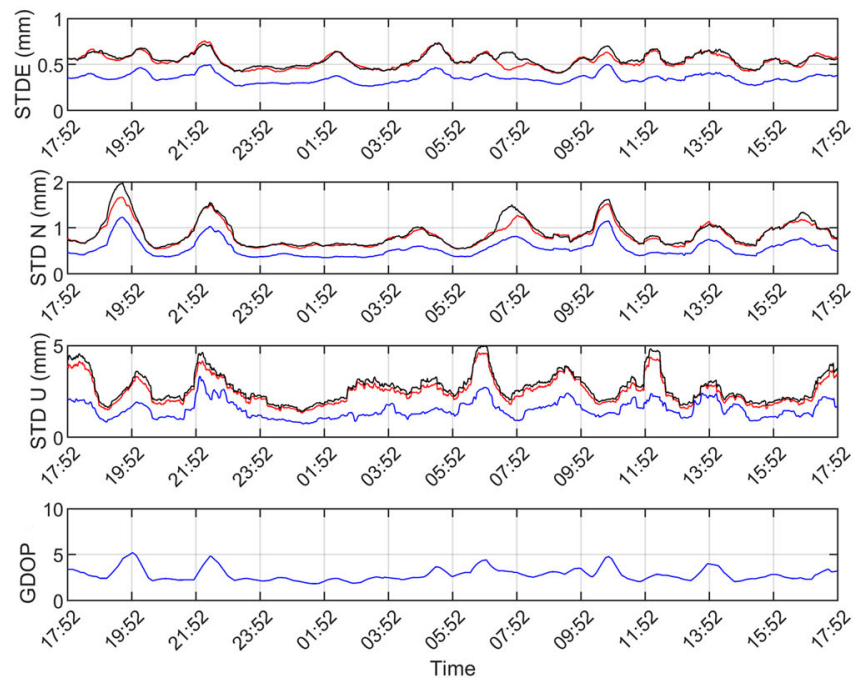

Fig. 5 The moving standard deviation (STD) of GPS-only time series for E/N/U and the corresponding GDOP moving average time series using (left) the patch antenna and (right) the geodetic antenna, having as baserover; both Leica receivers (blue line), both u-blox receivers (red) and
(Msaewe et al. 2017). The GNSS measurements of the four receivers were combined forming different base-rover couples and processed using DD mode. The same process was applied for each base-rover combination: kinematic processing mode with continuous ambiguity resolution, Saastamoinen troposphere model, and broadcast ionosphere model. The output of the processing was the time-series of the baseline length

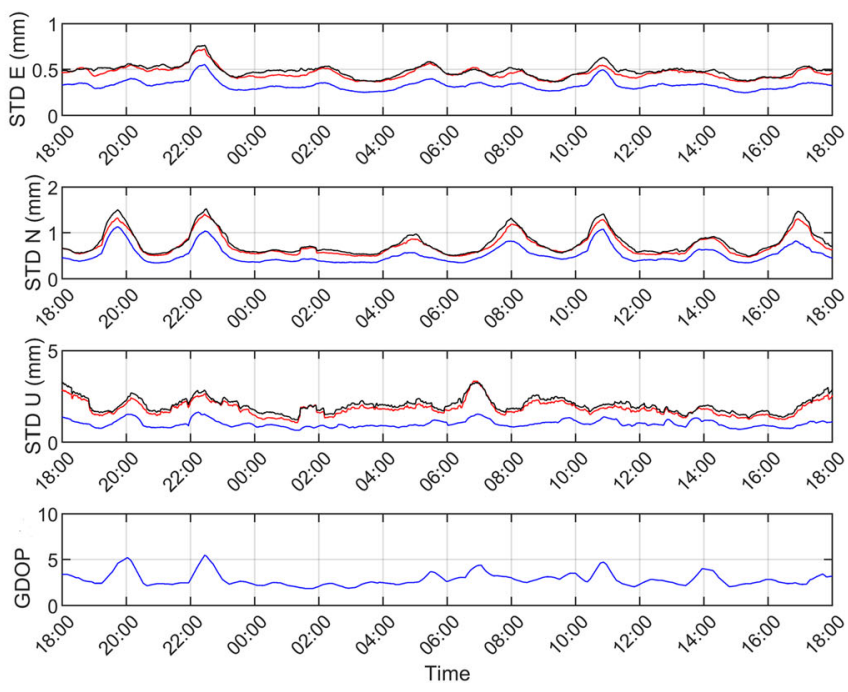

Leica (base) and u-blox (rover) receiver (black line).The precision of the GNSS time-series reflects the GDOP variance. It is evident the impact of the antenna in the precision of the Up component 


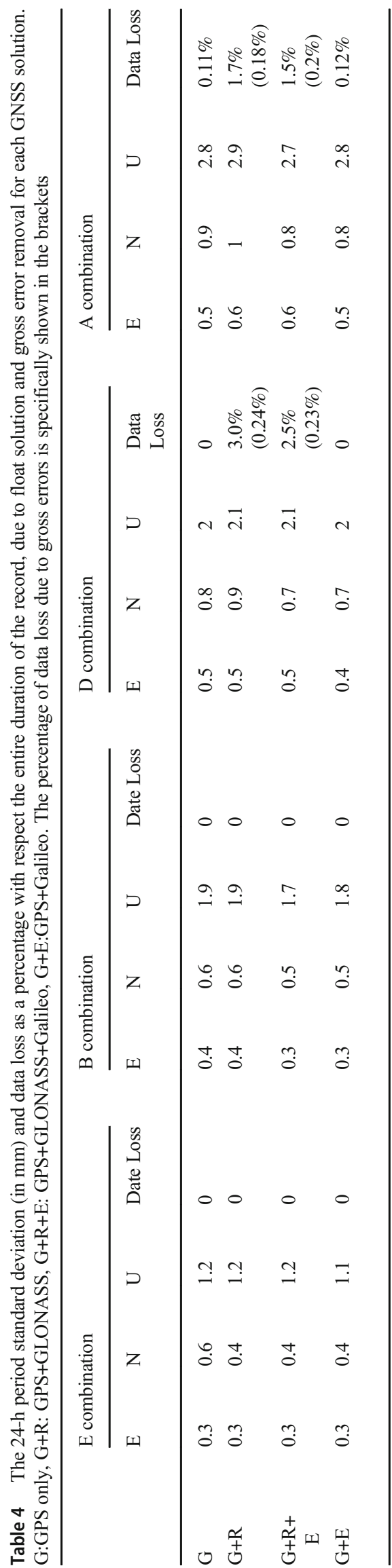

in Northing, Easting, and Up component. For ZBL measurements, the distance between base and rover receiver is zero. Therefore, the E,N,U time-series are with respect the baseline length, range around zero, and express the measurements noise.

There were examined three combinations of base-rover formation: (i) base and rover: u-blox receiver, (ii) base and rover: Leica receiver and (iii) base: Leica receiver and rover: u-blox receiver. Furthermore, since GPS, Galileo and GLONASS satellites signals were recorded, four different combinations of satellite constellation were examined: (i) GPS-only, (ii) GPS+GLONASS, (iii) GPS+GLONASS+ Galileo, and (iv) GPS+Galileo, to evaluate the contribution of multi-GNSS constellations on the performance of lowcost receiver. However, to achieve ambiguity resolution for the GLONASS measurements, the same receivers for base and rover are required due to Inter-Frequency Bias (IFB) (Al-Shaery et al. 2013); hence, for the combination Leica as base and $u$-blox as rover, only the GPS-only and GPS+Galileo solution were produced.

The 2-day experiments using the two types GNSS antennas resulted to six combinations of solutions (Table 1). The comparisons of the solutions between the different combinations show the effect of the different quality between the receivers and antennas (combinations A, B, D, and E). It was also evaluated the effect of using a low-cost receiver or geodetic receiver as base-reference (combinations A, C, D, and F).

\section{Zero-baseline measurement results}

The analysis of the zero-baseline measurements was applied on the E,N and Up coordinate time series of all the solutions. In Figs. 2 and 3, the E,N,U coordinate time-series of combination D (u-blox as base and rover receiver with geodetic antenna) and A (u-blox as base and rover receiver with patch antenna) for the four different satellite constellations are presented. Additional time series could also be found for the other combinations in supplementary material (Figures S1-S4). A preliminary analysis of the time series reveals the variation of their range which is due to the impact of the geometry of the current satellite constellation and the influence of the satellite systems; the GPS-only solution seems to have similar precision with the GPS+Galileo, as it is observed also from the mean and standard deviations of the time-series (Table 2 and 3), apart from few time periods (e.g., 22:00-23:00 or 10:00-11:00), where the GPS satellite constellation is weak. Regarding the GPS+GLONASS solution, it has reduced precision with periods of outliers, more significant in the Up component, which will be further analyzed. The GPS+GLONASS+Galileo solution seems to have similar performance to the GPS solution, apart from the periods of outliers which are produced due to the GLONASS constellation. Furthermore, the standard error computed for each epoch 
Fig. 6 Ratio of standard deviation between Leica - to - u-blox for each epoch for $\mathrm{E}, \mathrm{N}, \mathrm{U}$, when using the geodetic antenna (blue line) and the patch antenna (red line) and the corresponding GDOP time series

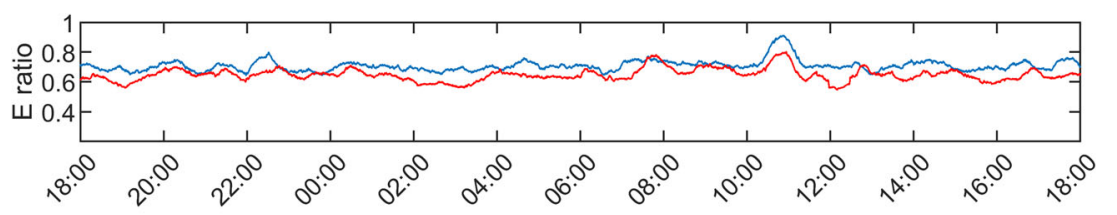

管 0.8 z 0.4

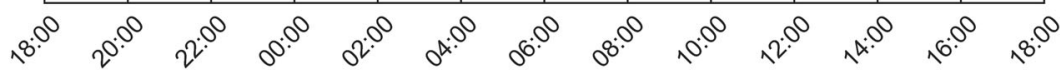
1
0.8

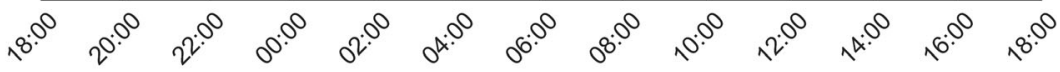

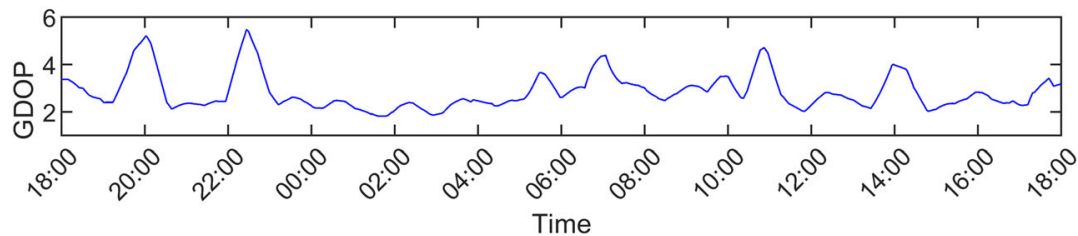

by RTKLib follows the same trend as the standard deviation of the time-series, having the lowest standard deviation for $\mathrm{E}$ component and then following $\mathrm{N}$ and $\mathrm{U}$ components. However, the standard error of the epochs characterized as outliers (spikes) do not have significant difference from that of the epochs with high precision.

Spectral analysis was also applied on the GNSS coordinate time-series using Discrete Fourier Transform (DFT) to identify noise characteristics of the GNSS measurements. In Fig. 4 are presented the spectra of combinations $\mathrm{A}, \mathrm{D}$, and $\mathrm{E}$ for GPS-only constellations, where it is observed that all three
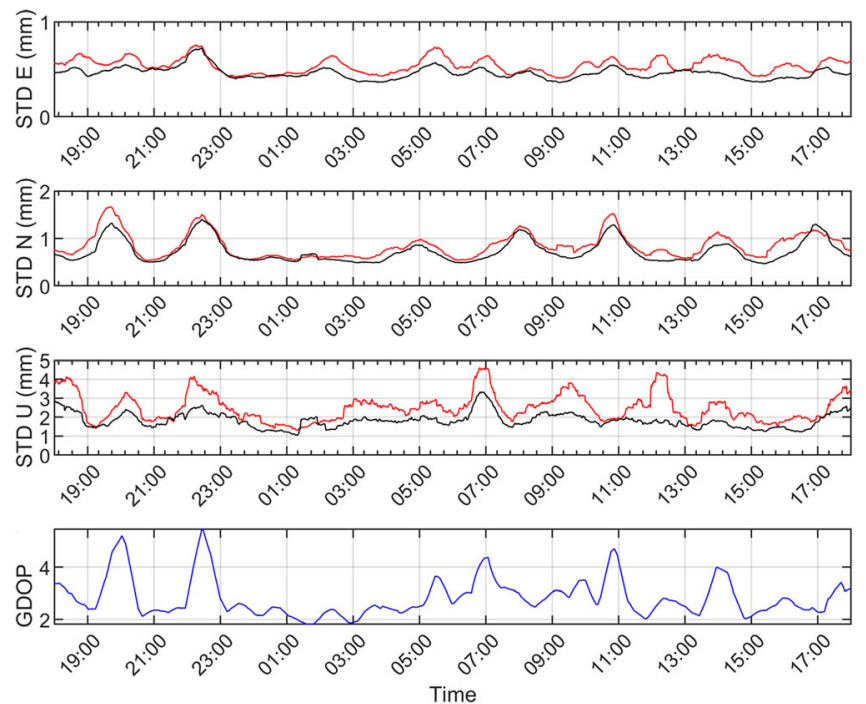

Fig. 7 Moving standard deviation (STD) of GPS-only coordinates timeseries for the combinations with patch (red line) and geodetic antenna (black line) for the ZBL measurements and having (left) u-blox receiver both as rover and base, and (right) Leica receiver both as rover and base. combinations have similar spectral characteristics for the horizontal components, with combination E (Leica as base and rover receiver with geodetic antenna) resulting to the least noisy spectrum mainly for low frequencies $(<0.01 \mathrm{~Hz})$. Furthermore, the spectra of the u-blox time-series appear similar characteristics regardless the antenna which is used (patch or AS10). Regarding the Up component, there is larger difference between the three spectra, with the combination $\mathrm{E}$ resulting again to the least noisy spectrum. Also, it is evident the impact of the quality of the antenna, as the spectrum of combination A (u-blox receiver-patch antenna) is the most
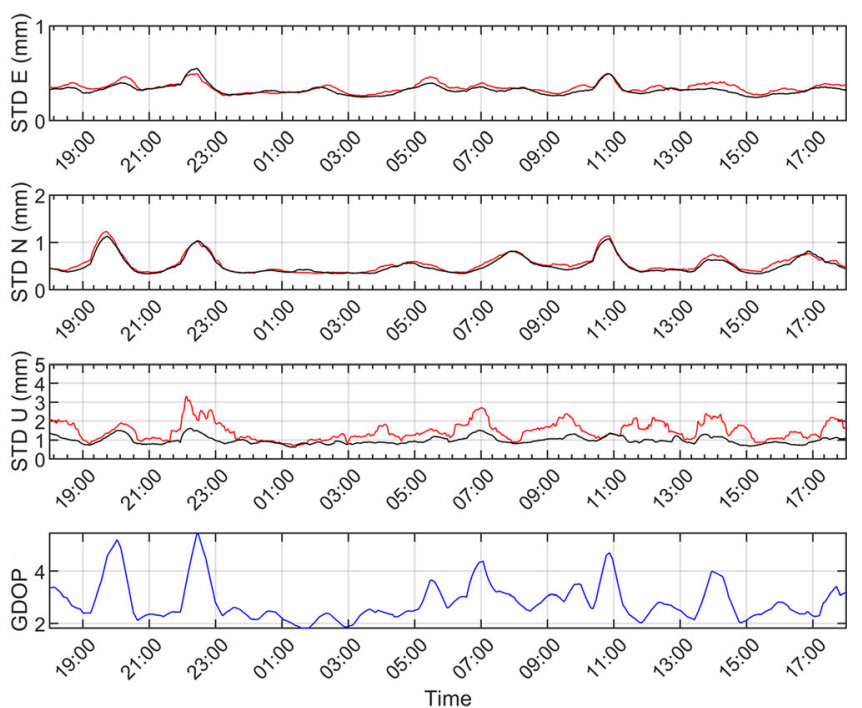

The E and N components generally follow the GDOP trend for both receivers. It is evident that the Up component is affected by the type of antenna 
Fig. 8 Moving standard deviation (STD) GNSS time-series of Leica receiver both as rover and base using the patch antenna with GDOP time series for all four multi-GNSS solutions (G:GPS, G+R:GPS+GLONASS; GPS+ Galileo:G+E; GPS+GLONASS+ Galileo:G+R+E). It is observed that the GPS solution generally follows the trend of GDOP, whereas the $\mathrm{G}+\mathrm{R}$ solution does not follow clearly the corresponding GDOP trend, especially for the E component
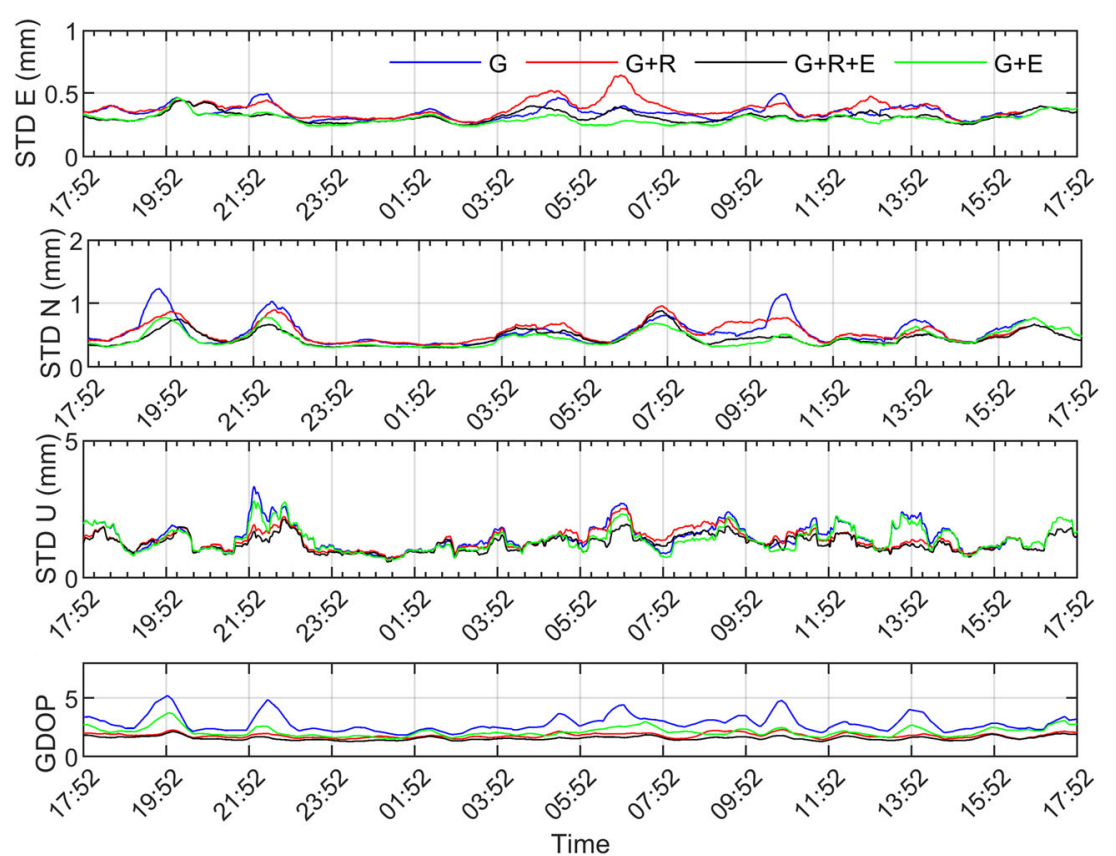

noisy especially for frequency larger than $0.01 \mathrm{~Hz}$, indicating that the level of the white noise band is amplified by the lowquality of the patch antenna. Spectra with similar results for GPS+GLONASS and GPS+GLONASS+Galileo are also shown in supplementary material (Figures S5-S7).

\section{Evaluation of the GNSS receiver performance}

To investigate the impact of the geometry of the satellite constellation on the performance of GNSS receivers, the GNSS coordinate time-series were correlated with GDOP (i.e., Geometric Dilution of Precision), which is the parameter describing position error due to satellite geometry. For ZBL measurements, the measurements noise is basically the result of the position error due to the satellite geometry amplified by the receiver noise. Hence, to analyze the performance of the two different GNSS receivers and the impact of the satellite constellation, the moving standard deviation (STD; timewindow of 1800s) of the coordinates time series was computed and correlated with the moving average of the GDOP time
Fig. 9 Moving standard deviation (STD) GNSS time-series of $\mathrm{u}$ blox receivers both as rover and base using the patch antenna with GDOP time series for all four multi-GNSS solutions. (G:GPS, G+R:GPS+GLONASS; GPS+ Galileo:G+E; GPS+GLONASS+ Galileo:G+R+E). Similar observations with Fig. 8; the G solution generally follows the trend of GDOP, whereas the G+R solution does not follow clearly the corresponding GDOP trend, especially for the E component
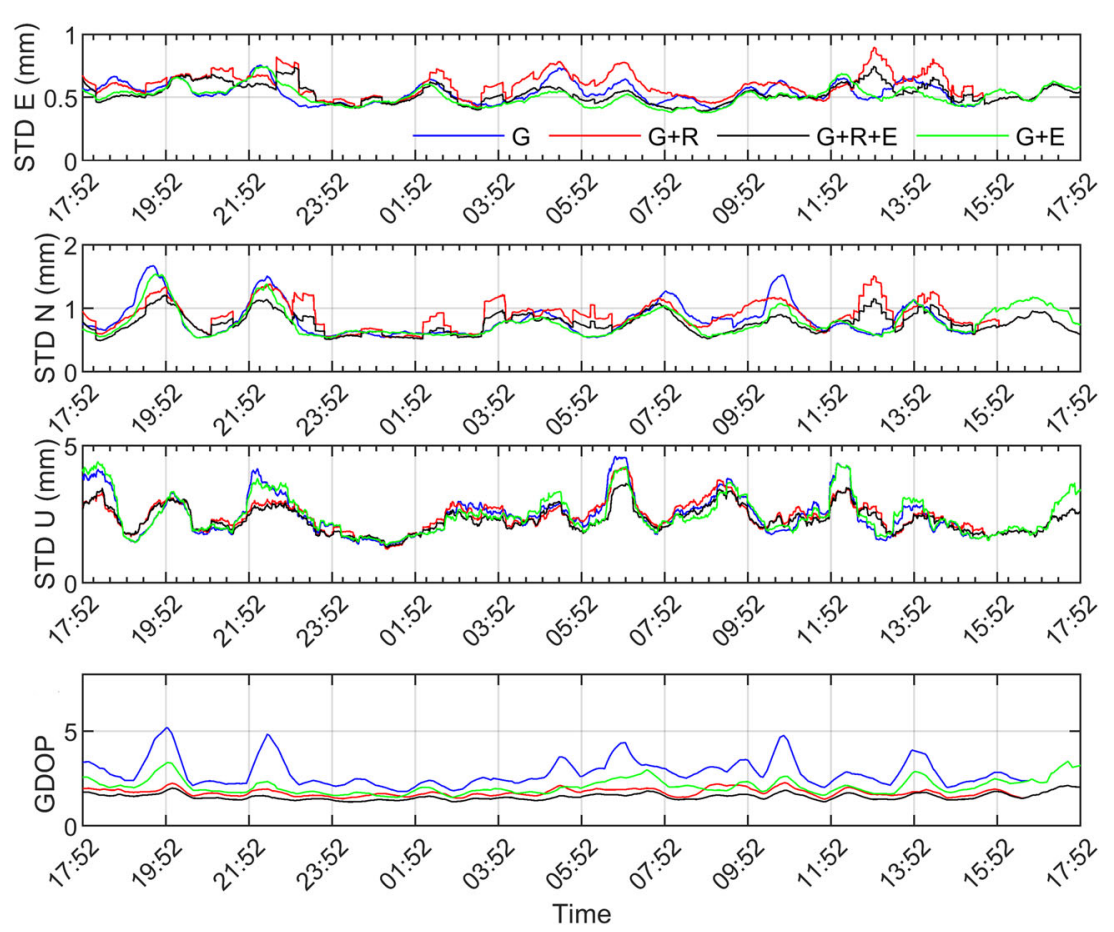
series (Fig. 5). The analysis of the ZBL time series indicates that the lowest standard deviation (i.e., lower noise) is achieved when the geodetic receivers are used both as the base and rover station (combinations B and E; Table 4), confirming the results of the spectral analysis (Fig. 4). Furthermore, the noise levels of the combinations $\mathrm{A}, \mathrm{C}$ and $\mathrm{D}, \mathrm{F}$, are at similar level. However, the combinations with u-blox receiver as base and rover (i.e. A and D) achieve slightly lower noise level. This is due to the different characteristics/function between $\mathrm{u}$ blox and Leica receivers, which may lead to different noise level of the GNSS time series especially for the zero-baseline measurements, where the receiver noise is dominant error source. Similar analysis is done for the GPS+Galileo solution for the different ZBL receiver grade combinations and provided in Figure S8. The GPS+GLONASS solution was not analyzed due to the impact of the inter-frequency bias.

Generally, the pattern of the moving standard deviation is similar for all the coordinate time series and in agreement with the GDOP time series, reflecting the impact of the GPS satellite constellation (Msaewe et al. 2017). For the horizontal components, there seems to be a constant difference of the noise level between the Leica $(\mathrm{B}, \mathrm{E})$ and $\mathrm{u}$-blox receivers $(\mathrm{A}, \mathrm{D})$, as expressed by the ratio between their moving standard deviation (Leica - to - u-blox), which fluctuates between 0.7 and 0.9 for $\mathrm{N}$ and $\mathrm{E}$ for geodetic antenna and $0.6-0.8$ for $\mathrm{N}$ and $\mathrm{E}$ for patch antenna, respectively, following the trend of the GDOP time series (Fig. 6). In both $\mathrm{E}$ and $\mathrm{N}$ component, the ratio of the geodetic antenna is higher than that of the patch antenna. Regarding the Up component, the difference of the noise level between the Leica and u-blox receivers varies more randomly, ranging from 0.4 to 0.9 , especially when the patch antenna is used. The correlation of the GDOP with the ratio of the Up component is not that clear, especially for the patch, indicating the subjectivity of the noise level to the antenna.

\section{Evaluation of the antennas performance}

The antenna' performance comparison is conducted through analysis of the moving standard deviation of combinations A, $\mathrm{C}$ and $\mathrm{D}, \mathrm{F}$ (Fig. 7). It is observed the highest precision in $\mathrm{E}$ component (sub-mm level) and then follows the $\mathrm{N}$ component (1-mm level); the small variations of moving standard deviation are dominantly related to the GDOP variations. However, the patch antenna had an additional impact on the measurement noise, especially for the $\mathrm{N}$ component, which increase the difference between the solutions of combinations A and D to $0.4 \mathrm{~mm}$ and $0.2 \mathrm{~mm}$ for $\mathrm{N}$ and $\mathrm{E}$, respectively, and makes the correlation between standard deviation and GDOP less strong, especially for relative low GDOP values (GDOP $<4$ ). Regarding the Up component, the GDOP is related strongly to standard deviation mainly for the combinations where geodetic antenna is used (combination A and C), whereas for the combinations where patch antenna is used (combination D and F), the patch antenna has significant impact on the measurements noise, as it is observed difference of the moving standard deviation between the combinations of geodetic and patch antenna reaching even up to $2.5 \mathrm{~mm}$ (see combinations $\mathrm{A}$ and D). The impact of the patch antenna on the vertical component is also confirmed by the spectral analysis in Fig. 4, where larger difference is noted for Up spectra than $\mathrm{E} / \mathrm{N}$ spectra. It is also seen that the high noise level is not always in agreement with the high GDOP values.

Also the ratio of Leica-to-u-blox noise is generally higher for the geodetic antenna than the patch antenna (Fig. 6), which also indicates the enhancement of the low-cost receivers precision due to the geodetic antenna. Regarding the vertical component, the ratio is higher for the patch antenna, especially

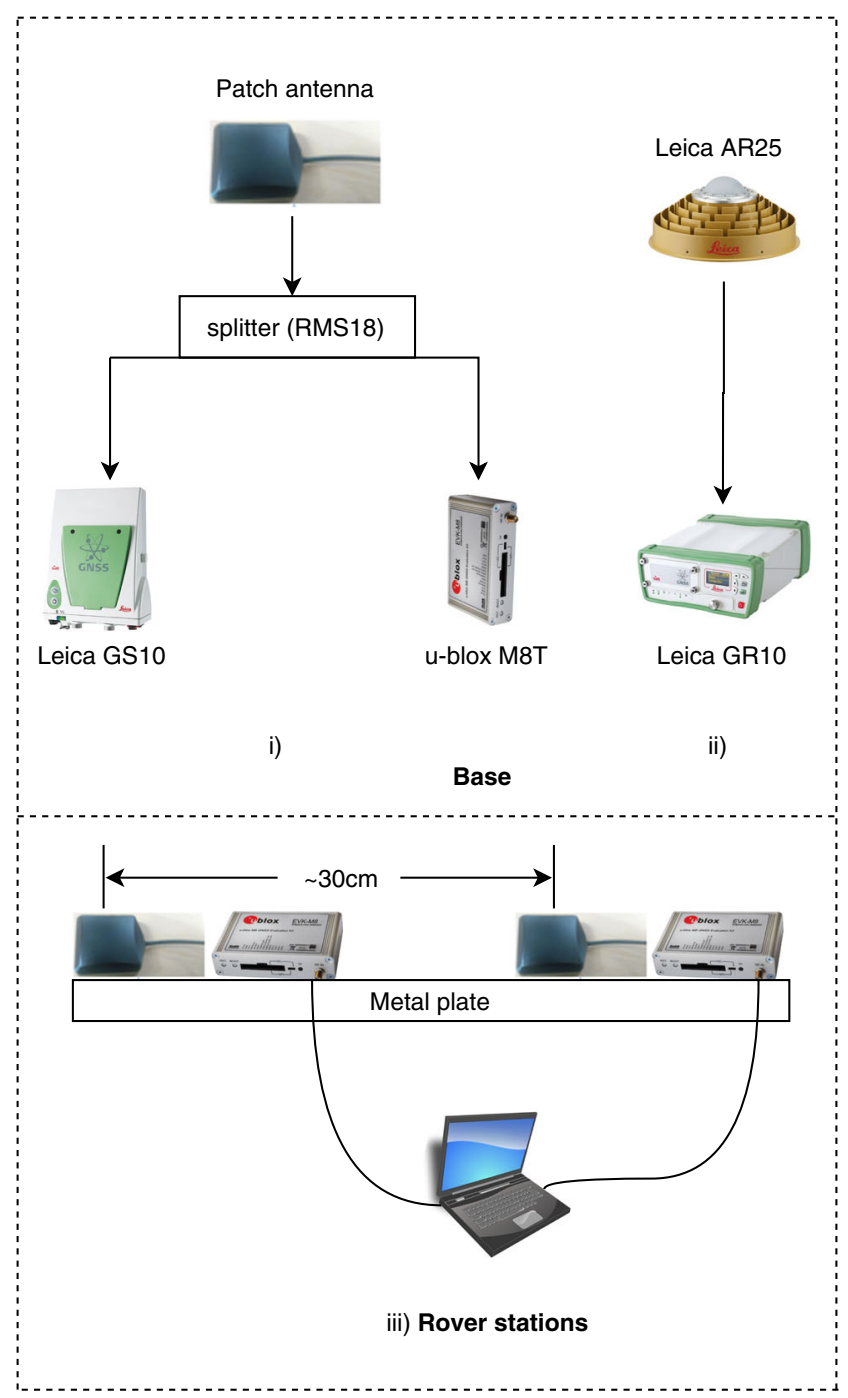

Fig. 10 The layout of the SBL measurements. Two different setups for base station: (i) patch antenna connecting to a geodetic and a low-cost receiver through signal splitter and (ii) choke-ring antenna connecting to geodetic grade receiver. The dual GNSS rover-system station consists of two closely spaced rover stations $(30 \mathrm{~cm}$ apart), with each using patch antenna connecting to $\mathrm{u}$-blox receiver 
Table 5 Different scenarios for the processing SBL results

\begin{tabular}{lllll}
\hline Combination & Base receiver & Base antenna & Rover receiver & Rover antenna \\
\hline G & Ubox M8T & patch antenna & u-blox M8T & patch antenna \\
$\mathrm{H}$ & GS10 & patch antenna & u-blox M8T & patch antenna \\
I & GR10 & Choke-ring antenna (AR25) & u-blox M8T & patch antenna \\
\hline
\end{tabular}

for the periods of poor constellation, which indicates the degradation of the Leica receiver precision due to the patch antenna.

\section{Evaluation of the multi-GNSS contribution}

Table 4 presents the overall standard deviation of the four GNSS solutions. For the performance of the Leica receivers, it is generally observed that the two multi-GNSS solutions, GPS+GLONASS+Galileo and GPS+Galileo, result to the best precision regardless the antenna which was used. However, for periods of good GPS satellite constellation, the achieved precision of GPS-only is practically the same with the multiGNSS solution (Figs. 8 and 9). Moreover, potential weak geometry of GLONASS satellite constellation or problematic function of GLONASS satellite could result to lower precision of multi-GNSS solution (for instance in Fig. 8, N component for the period 07:30-08:00), which was also proved by Msaewe et al. (2017).

Focusing on the u-blox receivers, it is also observed that the highest precision is achieved by the GPS+Galileo, while with the solutions including the GLONASS satellites (GPS+ GLONASS and GPS+GLONASS+Galileo) suffer from frequent outliers, as observed in Figs. 2 and 9. Those outliers are the result of cycle slips occurring for the GLONASS

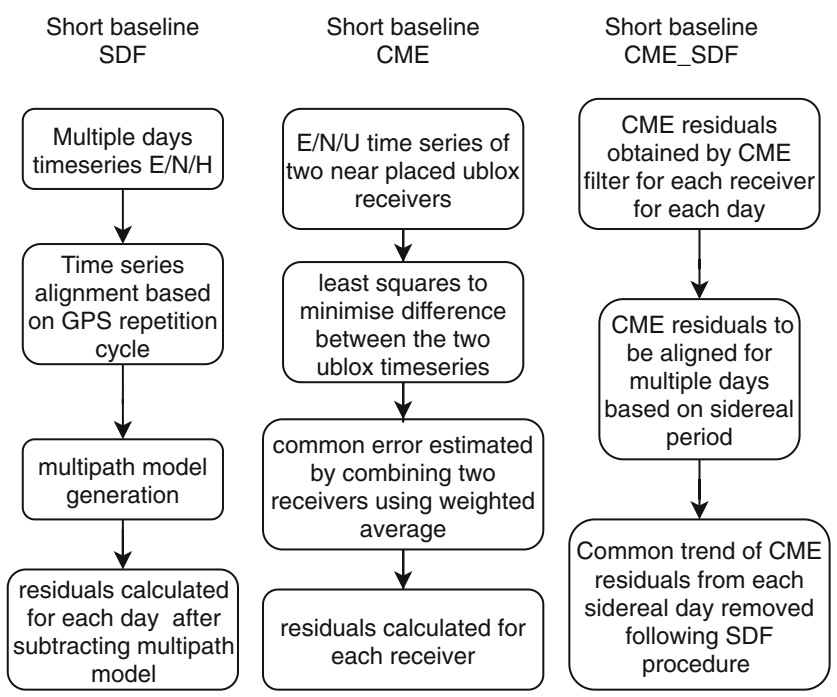

Fig. 11 Flow diagram of methodology/procedures for the application of sidereal filtering (SDF), common mode error (CME), and their combination. satellites in the u-blox measurements. These outliers seem not to depend on (i) the type of the antenna as they occur regardless which antenna was used, or (ii) the processing software, as the cycle slips occur also in the solution derived from Leica Infinity. The majority of these cycle slips seem not to occur in the Leica measurements. This is due to the fact that the SNR of the satellites which cause the cycle-slip are rejected by Leica receivers, while they are accepted by ublox receivers. On the contrary, the addition of Galileo system in the GNSS solution seems to enhance the precision of the GPS+Galileo solution and reduce the data gaps in the time series solution

\section{Short-baseline GNSS experiment}

\section{Short-baseline GNSS measurement}

Based on the findings of ZBL test, the short-baseline experiment was deployed, where the main aims were to evaluate the impact of the type of base GNSS station (geodetic receiverantenna or low-cost receiver and patch antenna) on the performance of the low-cost GNSS station and to investigate the potential enhancement of the performance of low-cost GNSS station by developing the dual low-cost GNSS system (i.e., deployment of two closely spaced low-cost GNSS rover stations) and applying the SDF and CME methods.

The experimental layout of SBL GNSS measurements is presented in Fig. 10. The dual GNSS rover-system station consists of two closely spaced low-cost GNSS stations, which are formed by two u-blox receivers and two patch antennas. The two patch antennas were orientated to the same azimuth and placed $30 \mathrm{~cm}$ distance apart in E-W direction, to avoid any signal interference and still retain similar multipath conditions. Both patch antennas were mounted on a large metal plate for multipath. Also, two base stations were set up: (i) one base station with a patch antenna, mounted on a metal plinth and connected via splitter to a Leica GS10 and a u-blox receiver and (ii) the second base station where a Leica GR10 receiver was connected to a Leica AR25 antenna. The measurements took place for three days (start: 16:04 06/08/2018 and end: 19:09 08/08/2018), where all the GNSS receivers were recording GPS, Galileo and GLONASS with $1 \mathrm{~Hz}$ sampling rate. The same type of GNSS receivers and antennas were used as in the zero-baseline measurements, to have consistency in the 
Fig. 12 The time-series of (i) u-blox2 E component GPS-only solution for three successive days of measurements, (ii) the multipath model based on the SDF, and (iii) the resultant residuals time series after subtracting the multipath error from the initial time-series. The precision of the residuals time-series reduces to $1-2 \mathrm{~mm}$ level. The application of the $\mathrm{N}$ and $\mathrm{U}$ time-series is presented in supplementary materials (Figure S9)

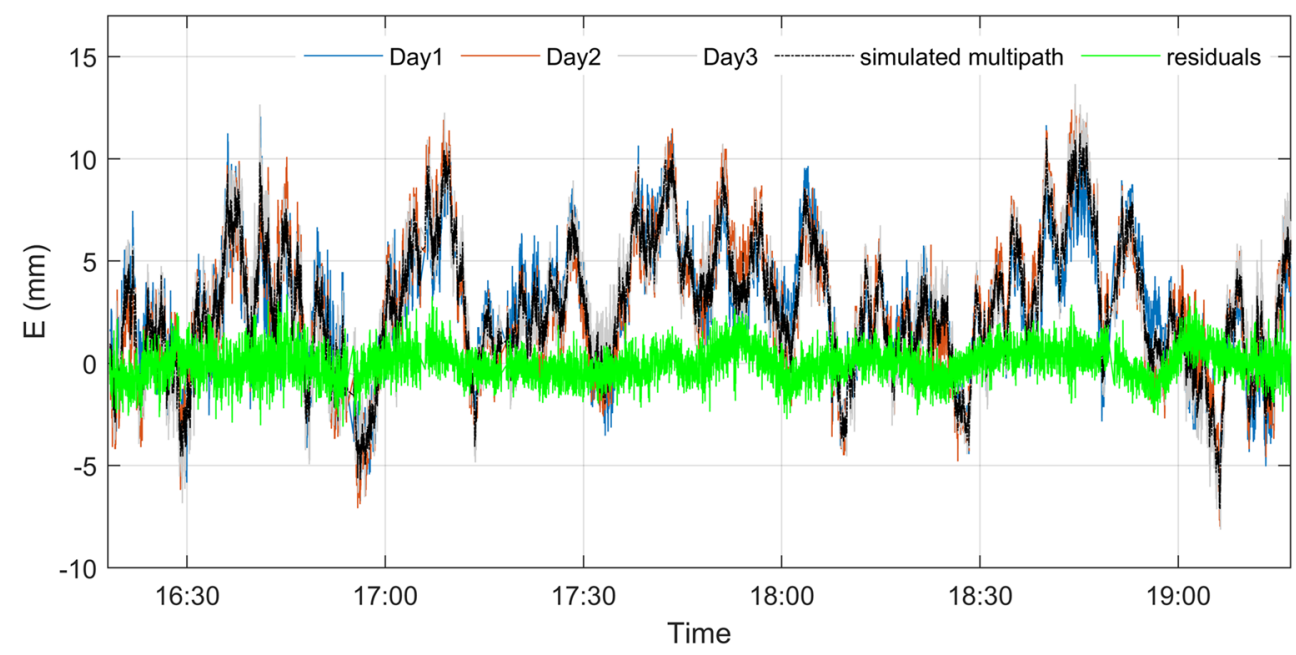

GNSS results. However, the high-grade GNSS station consisted of choke-ring antenna (Leica AR25) was used to evaluate potential impact of the antenna grade on the performance of the low-cost receivers.

\section{GNSS data processing and methodology of time-series analysis}

The GNSS data processing mode and configuration of ZBL was also used for SBL GNSS measurement to obtain the initial results time-series. In Table 5 are listed the short-baseline formations which were processed; each one resulted to two GNSS solutions for the two low-cost GNSS rover stations. Similar to the ZBL measurements, the GPS-only and GPS+ Galileo solutions were processed for all the three shortbaseline formations, while the GPS+GLONASS+Galileo solution was processed only for the formation with u-blox as base and rover. The combinations $\mathrm{H}$ and I were compared against combination $\mathrm{G}$ to evaluate the impact of the receiver and the antenna of the base station, respectively, on the performance of the low-cost GNSS station.
The SDF and CME methods were added in the analysis of the GNSS SBL time-series to investigate potential enhancement of the two closely spaced low-cost receivers precision by adopting the two mitigation error approaches. The SDF is a technique to remove constellation orbit related error, such as multipath or phase-center variation antenna error (Schmid et al. 2007), which depends strongly on the period of the full satellite orbit for the satellite system (Ragheb et al. 2007). The periodic orbit of the GPS satellites in their trajectories results in each satellite to appear at the exact same position about 4 min earlier from the previous sidereal day, defining the main principle of SDF which is that the relative geometry between the satellites and the antenna repeats between successive sidereal days with a time lag. Hence, after aligning the time series of consecutive days according to the period of the satellites orbit, the periodic errors related to the satellite constellation can be modelled by the "stacked" time-series. The SDF technique can be also applied to Galileo and GLONASS constellation solutions but not on the GNSS coordinates due to the different orbits of the systems satellites (10 and 8 sidereal days, respectively; Eissfeller et al. 2007). The calculation of
Fig. 13 The time-series of (i) u-blox1 and u-blox2 E GPS-only solution for Day 2, (ii) the CME model based on the time-series of two u-blox receivers, and (iii) the resultant residual time series after subtracting the CME model from the initial time-series. The residuals time-series follow a low-frequency signal trend and its range is at mm-level. The application of the $\mathrm{N}$ and $\mathrm{U}$ time-series is presented in supplementary materials (Figure S10)

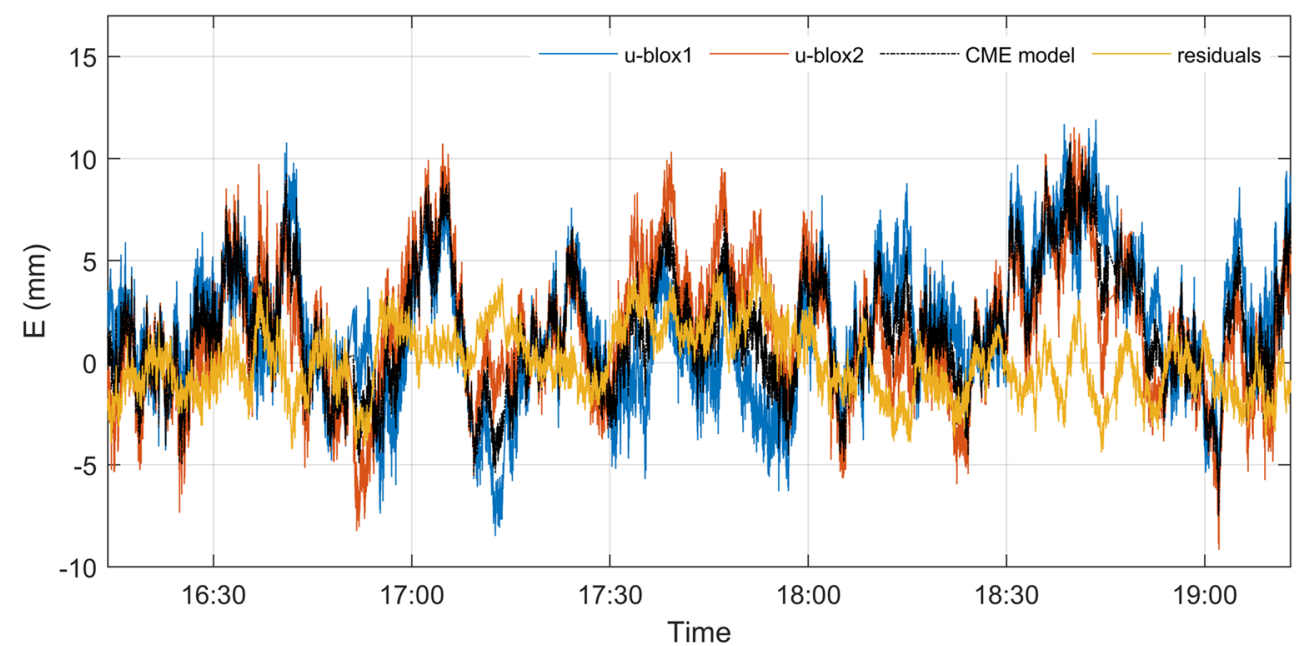


the model describing the SDF for each component (E,N, U) is given by the following equation:

$S D F \_E_{i}=\frac{\sum_{j=0}^{n-1} E_{(i-j * T)}}{n} S D F \_N_{i}=\frac{\sum_{j=0}^{n-1} N_{(i-j * T)}}{n} S D F_{-} U_{i}=\frac{\sum_{j=0}^{n-1} U_{(i-j * T)}}{n}$

where $i$ stands for current epoch, $j$ stands for number of days with respect the current day, $T$ stands for optimal lag, and $n$ is the total number of days stacked. The optimal sidereal period of $23 \mathrm{~h} 55 \mathrm{~m} 55 \mathrm{~s}$ was used for data alignment in this study to improve the precision at lower frequencies (Choi et al. 2004).

The CME technique is based on the assumption that the GNSS records of closely spaced stations are spatially correlated and include partly common errors. The CME error computation is based on the weighted average of the residual timeseries, also known as weighted stacking expressed by the equation below (Nikolaidis 2002):

$C M E_{t}^{i}=\frac{\sum_{j=1}^{n} \frac{R_{t}^{i}}{\sigma_{i}^{2}}}{\sum_{j=1}^{n} \frac{1}{\sigma_{i}^{2}}}$

where $C M E_{t}^{i}$ is the common-mode error at station $i$ at time $t$, $R_{t}^{i}$ is the coordinate time series for the station $i$ at time $\mathrm{t}, \sigma_{i}^{2}$ is the inverse of the square of the RMS of the station coordinate and $n$ is the number of stations. In our experiments, the two closely spaced u-blox receivers were $30 \mathrm{~cm}$ apart, receiving the satellite signals under the similar observation conditions, with main difference the slightly shifted multipath environment between the two GNSS stations. The latter makes CME method less effective than the SDF for the mitigation of multipath induced errors. However, the main advantage of CME against the SDF is that, it does not require multiple days of recording to apply the CME method and it is applied on coordinates time-series, making it applicable for multi-GNSS data. The CME method has been applied successfully for geodetic grade receivers in GNSS networks (Habboub et al. 2020), as it is unlikely to have closely spaced (in m-range) geodetic-grade receivers due to their high cost. Hence, the application of CME in closely spaced GNSS receivers, as examined in this study, is practically feasible only for lowcost GNSS receivers.

\section{Short-baseline measurement results}

The process of the GNSS measurements of the two u-blox receivers, following the same configuration as that of ZBL measurements, resulted in the $\mathrm{E}, \mathrm{N}, \mathrm{U}$ coordinate time-series with respect the current base station. During the recording period, the GNSS stations were stable, so any variation of the GNSS time-series expresses noise. The application of SDF, CME methods, and their combination followed the steps
Table 6 Mean average and standard deviation of E component GPS time-series for the 3 days measurements and after the application of the SDF method

\begin{tabular}{lllll}
\hline & Day1 & Day2 & Day3 & Residuals by SDF \\
\hline Mean average (mm) & 2.7 & 2.7 & 2.7 & 0 \\
Standard deviation (mm) & 3.1 & 3.3 & 3.3 & 0.8 \\
\hline
\end{tabular}

presented in Fig. 11. For the application of the SDF, the GPS time-series of the three days were aligned and stacked using Eq. 1, where the common time period (from 16:20 to 19:20) was used to model the multipath induced error to each of the two low-cost GNSS rover stations (Fig. 12). For the CME method, the GNSS time-series of the two low-cost GNSS receivers of each day were used to define the common error between the two stations using the Eq.2 (Fig. 13). For both SDF and CME, the modelled error was subtracted from the GPS/GNSS time-series to refine the GPS/GNSS time-series precision. For the combination of CME and SDF methods, the GPS time-series derived from CME method for the 3 days are then subjected to the SDF method, following the steps of SDF method. In Tables 6 and 7 are presented the mean average and the standard deviation of the time-series derived after the application of SDF and CME methods, respectively. It can be observed that the precision of the GPS and GNSS time-series are both significantly improved after the application of SDF and CME, as the standard deviation has been reduced to 0.8 and $1.7 \mathrm{~mm}$ for GPS and GNSS solution, respectively.

\section{Evaluation of the impact of the GNSS base station on low-cost GNSS rover performance}

In Fig. 14 are presented the moving standard deviation (15 min moving window) of the $E$ component time series of the u-blox 2 receiver, using as base station the three different available base station (low-cost GNSS station, Leica receiver with patch antenna, Leica receiver with AR25). The GPS-only and GPS+Galileo solutions were produced as the GLONASS satellites could not be included due to the inter-system bias between u-blox and Leica receivers. It is observed that there is no significant improvement by using Leica receiver instead of u-blox, for both GPS-only and GPS+Galileo solutions, when

Table 7 Mean average and standard deviation of E component GPS time-series for the $\mathrm{u}$-blox stations of Day 2 and after the application of the CME method

\begin{tabular}{llll}
\hline & u-blox1 & u-blox2 & Residuals by CME \\
\hline Mean average (mm) & 1.5 & 1.5 & 0 \\
Standard deviation (mm) & 3.3 & 3.3 & 1.7 \\
\hline
\end{tabular}




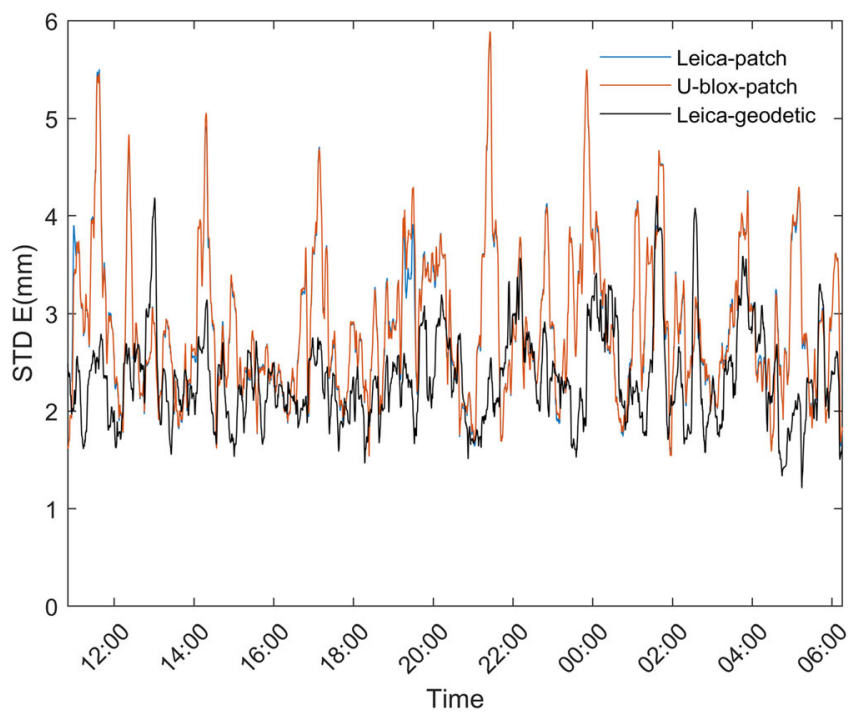

Fig. 14 The time-series of moving standard deviation (STD) of the $u$ blox2 E components of Day2 by using as base stations (i) u-blox with patch antenna, (ii) Leica receiver with patch antenna, and (iii) Leica receiver with geodetic antenna, derived from (left) GPS-only solutions

patch antenna is used for the base station. However, for the base station with Leica receiver and antenna, there is significant improvement in the precision of the low-cost receiver, especially for the GPS-only solution, which reaches even $3 \mathrm{~mm}$ for the $E$ component.

Furthermore, by applying SDF and CME method, there is significant improvement on the low-cost receiver precision, which is reduced below $2 \mathrm{~mm}$ for both methods (Fig. 15). For the SDF method, the same accuracy is achieved regardless the receiver type (geodetic or low-cost) when connected to patch antenna. However, there is improvement in the precision, reaching up to $0.4 \mathrm{~mm}$, when using a geodetic antenna

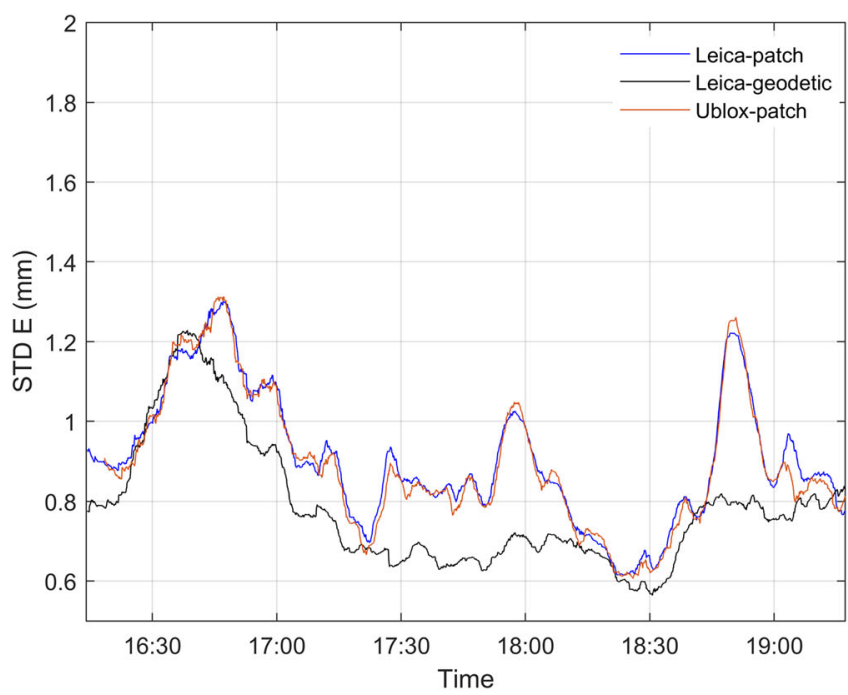

Fig. 15 The time-series of moving standard deviation (STD) of the ublox2 E component of Day2 after using (left) SDF and (right) CME analysis when there are used as base stations (i) u-blox with patch antenna, (ii) Leica receiver with patch antenna, and (iii) Leica receiver

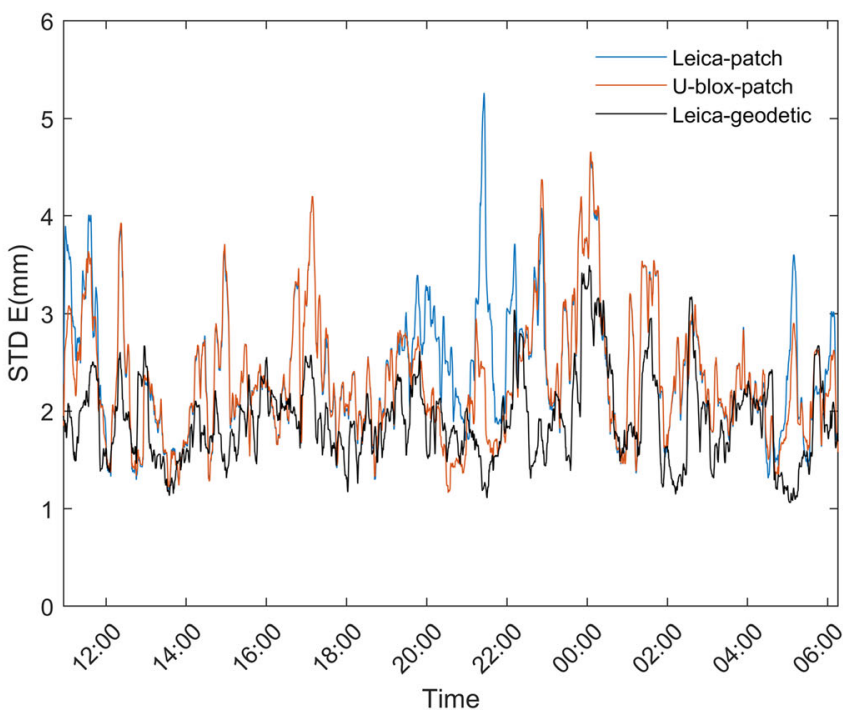

and (right) GPS+Galileo solutions. The solution, having as base Leica receiver and geodetic antenna, has the lowest STD, whereas the solutions with u-blox at base station has the same STD regardless the antenna which is used at the station

(AR25) at the base station. Regarding the application of the CME method, the same precision is achieved regardless the type of the GNSS antenna and receiver of the base station, as the potential errors, introduced by the receiver and/or antenna of the base station, are limited through the CME method of the two closely spaced low-cost stations. Finally, it is also observed that the SDF method can achieve higher precision than that of CME method with the GPS only results.

The GNSS N and U time-series were subjected in the same analysis and led to the same observations (in the supplementary materials Figures S11-S12).

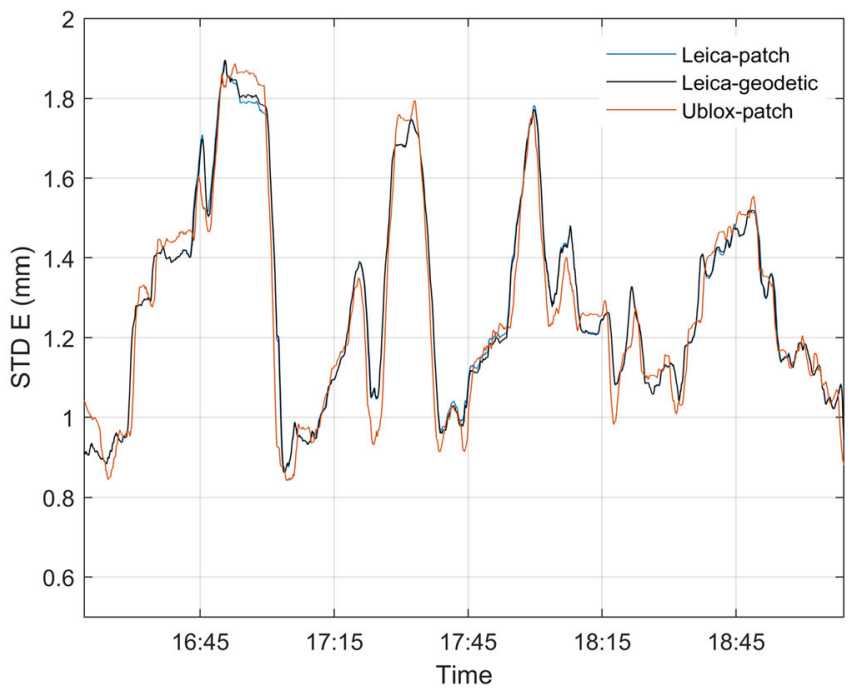

with geodetic antenna for GPS only constellation. The SDF lead to lower STD for the solution using as base Leica receiver with geodetic antenna, whereas for the CME all three solutions have the same level of STD 
Fig. 16 The time-series of the moving standard deviation (STD) of the u-blox 2 coordinate time series of Day2 for the solutions using GPS only (G), GPS+ Galileo (GE) and GPS+ GLONASS+Galileo (GRE), and the sidereal filtered (SDF) residuals with GPS only constellation. The SDF time-series has generally the lowest STD for all the components from any other GNSS solution
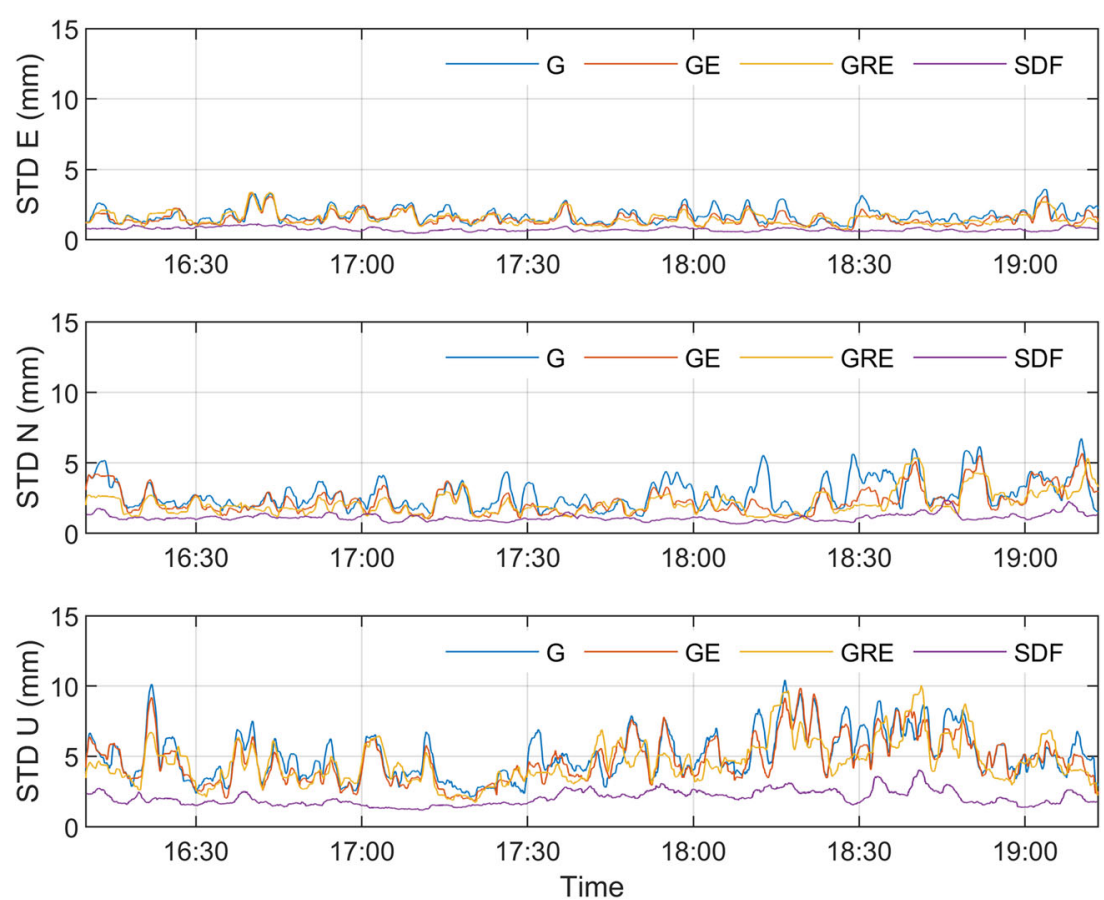

\section{Evaluation of the performance of the single low-cost multi-GNSS station}

The GNSS time series of the two low-cost GNSS receivers were analyzed and the moving standard deviation was computed for the 3 days. In Fig. 16 are presented the moving standard deviation of the Day2 u-blox 2 coordinate time series between 16:20 and 19:20 of the GPS-only, GPS+Galileo, and GPS+GLONASS+Galileo solutions. It is obvious that for most of the time period, the two multi-GNSS solutions lead to higher precision than the GPS-only solution. By comparing the moving standard deviation of the multi-GNSS solutions against the GPS-only solution of the low-cost GNSS u-blox2 receiver, it was computed the time-period that the multiGNSS solutions are of higher precision as percentage of the examined period (Table 8). It is clear that the GPS+Galileo solution leads to higher precision than the GPS-only solution for more than $70 \%$ of the examined time periods, especially for Northing and Up component. The precision is even higher when the GLONASS constellation is included, as the multiGNSS solution gives better precision than the GPS-only solution for the $75 \%$ of the period. Similar were the results based on the analysis of the u-blox1 receiver data.

However, the multi-GNSS solutions resulted to more gaps, which could correspond to float or even no solution. More specifically, for the low-cost u-blox2, the GPS-only solution has the highest availability for all the days reaching up to $99 \%$, while the GPS+Galileo solution leads to slightly lower availability with $97 \%$ of the GNSS recording period. The GLONASS constellation reduces the availability of the multi-GNSS solution for the low-cost GNSS receiver, dropping it at the $86 \%$ of the recording period. As it was mentioned for the ZBL measurements, this is the result of the cycle slips produced by GLONASS satellites in u-blox receivers, which is limited in the records of other GNSS receivers (for instance Leica GS10), as the poor quality of the signal of the problematic GLONASS satellite(s) is rejected.

Furthermore, the application of SDF in the GPS-only solution of the low-cost GNSS receivers for the common period led to the significant reduction of the noise level of the GPSonly solution, which is significantly lower even than any of the multi-GNSS solution. For instance, the moving standard
Table 8 Comparison between the moving standard deviation (STD) of u-blox2 coordinate time series for the three GNSS solutions (GPS only, GPS+GLONASS, GPS+Galileo). The comparison is expressed as percentage with respect the examined period

\begin{tabular}{|c|c|c|c|c|c|c|}
\hline \multirow[t]{2}{*}{ Moving STD (\%) } & \multicolumn{3}{|c|}{$G E$ smaller than GPS-only } & \multicolumn{3}{|c|}{$G R E$ is smaller than GPS-only } \\
\hline & $\mathrm{E}$ & $\mathrm{N}$ & $\mathrm{U}$ & $\mathrm{E}$ & $\mathrm{N}$ & $\mathrm{U}$ \\
\hline Day1 & $68.9 \%$ & $80.7 \%$ & $68.8 \%$ & $78.5 \%$ & $85.2 \%$ & $87.9 \%$ \\
\hline Day2 & $78.7 \%$ & $79.5 \%$ & $81.7 \%$ & $78.2 \%$ & $87.6 \%$ & $86.8 \%$ \\
\hline Day3 & $69.3 \%$ & $75.0 \%$ & $83.9 \%$ & $77.7 \%$ & $93.0 \%$ & $91.7 \%$ \\
\hline
\end{tabular}


Table 9 Comparison of the moving standard deviation of the u-blox2 coordinates time-series derived after the application of SDF against all the multi-GNSS u-blox 2 coordinate time series. The comparison indicates whether the STD of the SDF time-series is smaller than that of the multi-GNSS solutions and it is expressed as percentage with respect the examined time period

STD of SDF smaller than STD of multi-GNSS (\%)

\begin{tabular}{llll}
\hline & $\mathrm{E}$ & $\mathrm{N}$ & $\mathrm{U}$ \\
\hline Day1 & $96.0 \%$ & $87.8 \%$ & $97.5 \%$ \\
Day2 & $100.0 \%$ & $92.2 \%$ & $96.3 \%$ \\
Day3 & $97.5 \%$ & $84.5 \%$ & $95.8 \%$ \\
\hline
\end{tabular}

deviation for the horizontal and vertical components of lowcost u-blox 2 receiver is lower than $2 \mathrm{~mm}$ and $4 \mathrm{~mm}$, respectively, as shown in Fig. 16. By comparing the moving standard deviation of the GPS SDF time-series with that of the multi-GNSS time-series, it is evident that the GPS SDF solution is more precise than any multi-GNSS solution for at least $85 \%$ of the recording time period (Table 9).

\section{Evaluation of the performance of the dual low-cost GNSS rover-system}

In Fig. 17 are presented the moving standard deviation of the of E,N, U component of the low-cost GNSS u-blox2 receiver for the different GNSS solutions and after the application of the CME method and it is compared against the corresponding moving standard deviation of the GPS-only solution after application of the SDF. It is observed that the CME multiGNSS solution of GPS+GLONASS+Galileo constellation is the most precise between the CME GNSS solutions, apart from time periods (e.g., 18:15-18:25 and 18:50-19:00) where the poor quality of GLONASS satellite(s) signal reduce the precision of the multi-GNSS. In Table 10 is presented the comparison between the achieved moving standard deviation of the three GNSS solutions after the application of CME and expressed as percentage with respect the examined time period. It is confirmed that, the CME GPS+GLONASS+Galileo time series is the most precise for $\sim 70 \%$ of the recording period for any of the three days. The smallest improvement of the precision is observed in the Easting component, observed also in Fig. 17, as high precision (i.e., $<2 \mathrm{~mm}$ ) is achieved by all CME GNSS solutions. This is probably due to the satellite constellation and also the deployment of the two low-cost GNSS rover stations, which had E-W direction baselines.

Furthermore, by comparing the moving standard deviation of the SDF GPS-only u-blox2 time series with the CME GNSS time series, it is obvious that the SDF GPS-only solution does not vary significantly, whereas the CME GNSS solutions vary especially for the time intervals around 18:00 or 19:00. However, by comparing the achieved precision for the entire time period, it was observed that the SDF GPS-only time series achieves lower moving standard deviation (higher precision) than the CME GPS-only time series for $\sim 60-80 \%$ of the time-series, depending on the component. On the contrary, the time series of the other two CME GNSS solutions (GPS+Galileo and GPS+GLONASS+Galileo) achieve similar
Fig. 17 Comparison of the moving standard deviation (STD) of the u-blox 2 coordinate time series of Day 2 derived after the application of SDF method for GPS-only solution and CME method for all the available multi-GNSS solutions (G: GPS-only; GE:GPS+Galileo; GRE:GPS+GLONASS+Galileo)
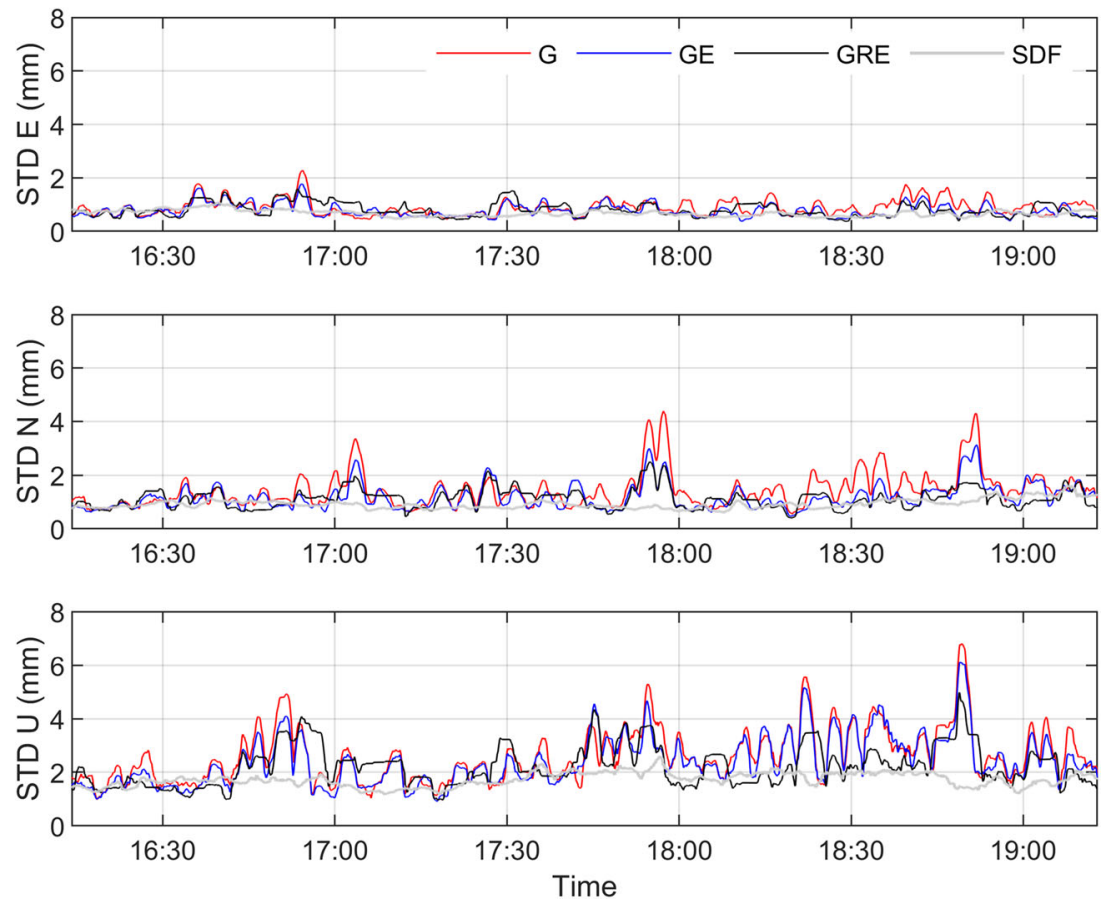
Table 10 Comparison between the moving standard deviation (STD) of the $\mathrm{u}$-blox 2 coordinate time-series after the application of CME for the three multi-GNSS solutions (G:GPS only, GE:GPS+Galileo GRE:GPS+
GLONASS+Galileo). The comparison is expressed in percentage of the examined period

\begin{tabular}{|c|c|c|c|c|c|c|c|c|c|}
\hline \multirow[t]{2}{*}{ STD $(\%)$} & \multicolumn{3}{|l|}{ Day1 } & \multicolumn{3}{|c|}{ Day2 } & \multicolumn{3}{|l|}{ Day3 } \\
\hline & $\mathrm{E}$ & $\mathrm{N}$ & $\mathrm{U}$ & $\mathrm{E}$ & $\mathrm{N}$ & $\mathrm{U}$ & $\mathrm{E}$ & $\mathrm{N}$ & $\mathrm{U}$ \\
\hline GRE lower than $G$ & $62 \%$ & $74 \%$ & $76 \%$ & $74 \%$ & $74 \%$ & $74 \%$ & $67 \%$ & $79 \%$ & $82 \%$ \\
\hline GE lower than $\mathrm{G}$ & $70 \%$ & $73 \%$ & $77 \%$ & $73 \%$ & $80 \%$ & $72 \%$ & $69 \%$ & $83 \%$ & $75 \%$ \\
\hline GRE lower than GE & $55 \%$ & $71 \%$ & $66 \%$ & $57 \%$ & $65 \%$ & $65 \%$ & $63 \%$ & $71 \%$ & $71 \%$ \\
\hline
\end{tabular}

or higher precision than the SDF GPS-only solution and especially the CME GPS+GLONASS+Galileo time series which can be more precise even up to $\sim 60-70 \%$ of the timeseries (Table 11).

The analysis of the CME GPS-only solutions for the common time-period of the 3 days shows that there is some repeatability in the pattern of their moving standard deviation, with a time lag of $\sim 4 \mathrm{~min}$, indicating potential presence of some multipath induced error in the solution (Fig. 18). Thus, to enhance even further the CME GPS-only solution, the SDF was applied in the CME GPS-only time-series. In Fig. 19 are presented the moving standard deviation of the SDF-CME GPS-only solution against the CME GPS-only solution, where it is observed that the precision is further improved in the horizontal components and dropping below $1 \mathrm{~mm}$, while for the Up component it is reduced below $2 \mathrm{~mm}$. Likewise, by applying first the SDF of the GPS-only solutions of the two low-cost GNSS rover stations and then the CME method, in order to remove any potential common error between the two GPS time-series, it led to similar precision indicating that the sequence of the application of SDF and CME in the GPS solutions of the two GNSS rover stations do not affect the achieved precision.

\section{Discussion}

By analyzing the results of the ZBL experiments, it is confirmed the higher noise level of the u-blox with respect the geodetic receivers (Leica), as it was revealed also from previous studies of Odolinski and Teunissen (2017) and Takasu and Yasuda (2008). The difference of the noise level did not exceed $0.3 \mathrm{~mm}$ and $0.8 \mathrm{~mm}$ for the horizontal and vertical components, respectively (Table 4). Also, application of patch antenna instead of geodetic seems to influence both receivers but mostly the u-blox receivers, especially for the vertical component, as the noise level of the $\mathrm{u}$-blox and Leica receiver increases by $0.9 \mathrm{~mm}$ and $0.7 \mathrm{~mm}$, respectively. Regarding the performance of the low-cost receivers for different satellite constellations, the multi-GNSS solutions have higher precision than the GPS-only solution mainly for the periods when the geometry of the GPS satellite constellation is relative poor; this is also verified for the performance of geodetic receivers (Msaewe et al. 2017). Furthermore, the most reliable multiGNSS solution is the GPS+Galileo solution, as the solutions which include observations from GLONASS satellites suffer from outliers, mainly produced by cycle slips due to weak GLONASS satellite(s) signal. This phenomenon is amplified by using patch antenna. However, this phenomenon is limited in Leica receivers because the geodetic receivers reject the poor satellite signal of the GLONASS satellites. Based on the findings above, the short-baseline experiment was carried out and it was observed that the low-cost GNSS rover station performs similarly when the base station adopts patch antenna, regardless the base station receiver grade (geodetic or lowcost). On the other hand, the performance of the low-cost GNSS rover station is improved by using base station consisted of geodetic receiver and geodetic antenna, reducing

GRE:GPS+GLONASS+Galileo). The comparison is expressed in percentage of the examined period
Table 11 Comparison of the moving standard deviation (STD) of the $\mathrm{u}-$ blox2 GPS-only SDF coordinates time-series and the three u-blox2 GNSS coordinate CME time-series (G:GPS only, GE:GPS+Galileo

\begin{tabular}{|c|c|c|c|c|c|c|c|c|c|}
\hline \multirow[t]{2}{*}{ STD $(\%)$} & \multicolumn{2}{|c|}{ SDF lower than G CME } & \multicolumn{4}{|c|}{ SDF lower than GE CME } & \multicolumn{3}{|c|}{ SDF lower than GRE CME } \\
\hline & $\mathrm{E}$ & $\mathrm{N}$ & $\mathrm{U}$ & E & $\mathrm{N}$ & $\mathrm{U}$ & $\mathrm{E}$ & $\mathrm{N}$ & $\mathrm{U}$ \\
\hline Day 1 & $58 \%$ & $63 \%$ & $69 \%$ & $41 \%$ & $48 \%$ & $48 \%$ & $38 \%$ & $38 \%$ & $44 \%$ \\
\hline Day 2 & $74 \%$ & $85 \%$ & $86 \%$ & $62 \%$ & $67 \%$ & $80 \%$ & $56 \%$ & $58 \%$ & $68 \%$ \\
\hline Day3 & $60 \%$ & $64 \%$ & $66 \%$ & $51 \%$ & $41 \%$ & $48 \%$ & $49 \%$ & $35 \%$ & $33 \%$ \\
\hline
\end{tabular}



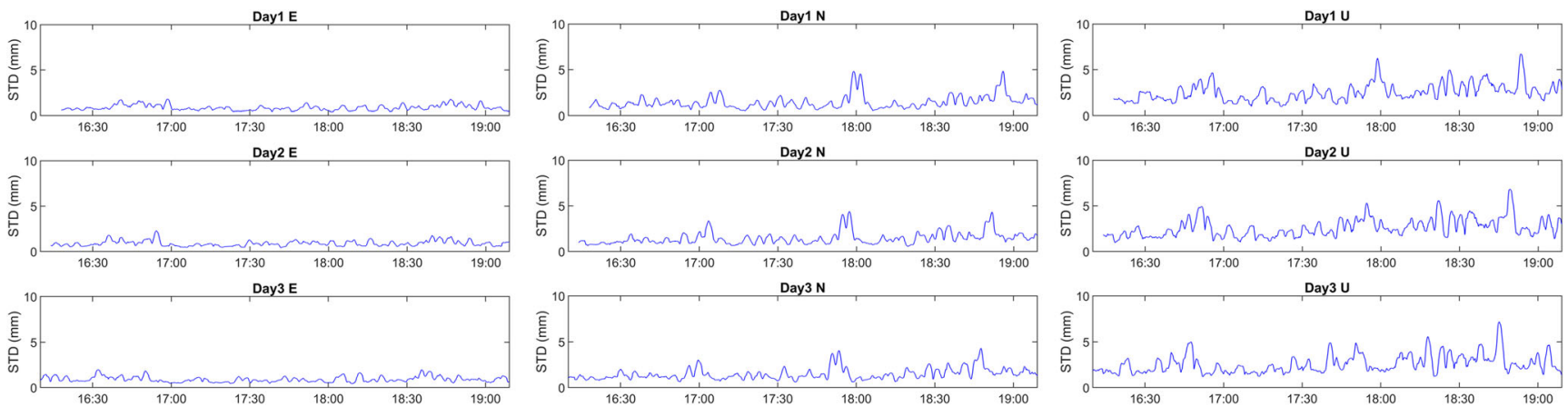

Fig. 18 The u-blox2 E,N,U GPS-only coordinates time-series after CME filtering for the three consecutive days. It is evident the repetition of some error anomalies (for instance $\sim 18: 00$ in Day 1), appearing with a time lag of $\sim 4 \mathrm{~min}$, indicating potential multipath induced errors

the noise level of $E$ component even to $2 \mathrm{~mm}$. The application of multi-GNSS solution, especially GPS+Galileo, can also enhance the performance of the rover low-cost GNSS station. However, the multi-GNSS solution seemed to have less availability, due to gaps and float solutions, which is amplified when using observations of GLONASS satellites. Another method of precision improvement is from SDF with GPSonly solution, where the application of SDF improves significantly the noise level of the GNSS rover station, especially when patch antenna is used also on the base station. The improvement in GPS time-series by applying SDF method is well-known for geodetic-grade receivers. However, for lowcost GNSS receivers, the SDF method probably have larger improvement in the precision due to the higher noise level of the low-cost GNSS receivers.

The above observation of ZBL and SBL measurements led to the formation of the dual low-cost GNSS receivers, using the two closely spaced low-cost multi-GNSS receivers in order to apply the CME method and improve the measurements precision. From the analysis of the different CME multiGNSS solutions, it was again revealed that the CME GPS+ Galileo solution proved the most reliable and generally more precise than the SDF GPS-only solution; however, the SDF GPS-only solution seems to have less variations of the amplitude of the time-series, especially for the vertical component. By applying the CME for the GPS-only solutions between the two GNSS stations to remove common error between the two receivers and then the SDF to limit the multipath -induced errors in each individually GNSS rover station, the highest precision could be achieved, which reaches $1 \mathrm{~mm}$ for the horizontal components and $2 \mathrm{~mm}$ for the vertical component.

Figure 20 presents the original time-series of short baseline GPS solution of low-cost GNSS receiver with low-cost GNSS base station (low-cost GNSS receiver and patch antenna), and
Fig. 19 The moving standard deviation (STD) of u-blox2 GPS-only coordinate time-series after the application of SDF (blue) and CME-SDF (red) for Day 1. It is evident that the application of CME and SDF method achieve higher precision than SDF only method, reaching up to $1 \mathrm{~mm}$ and $2 \mathrm{~mm}$ for horizontal and vertical components, respectively
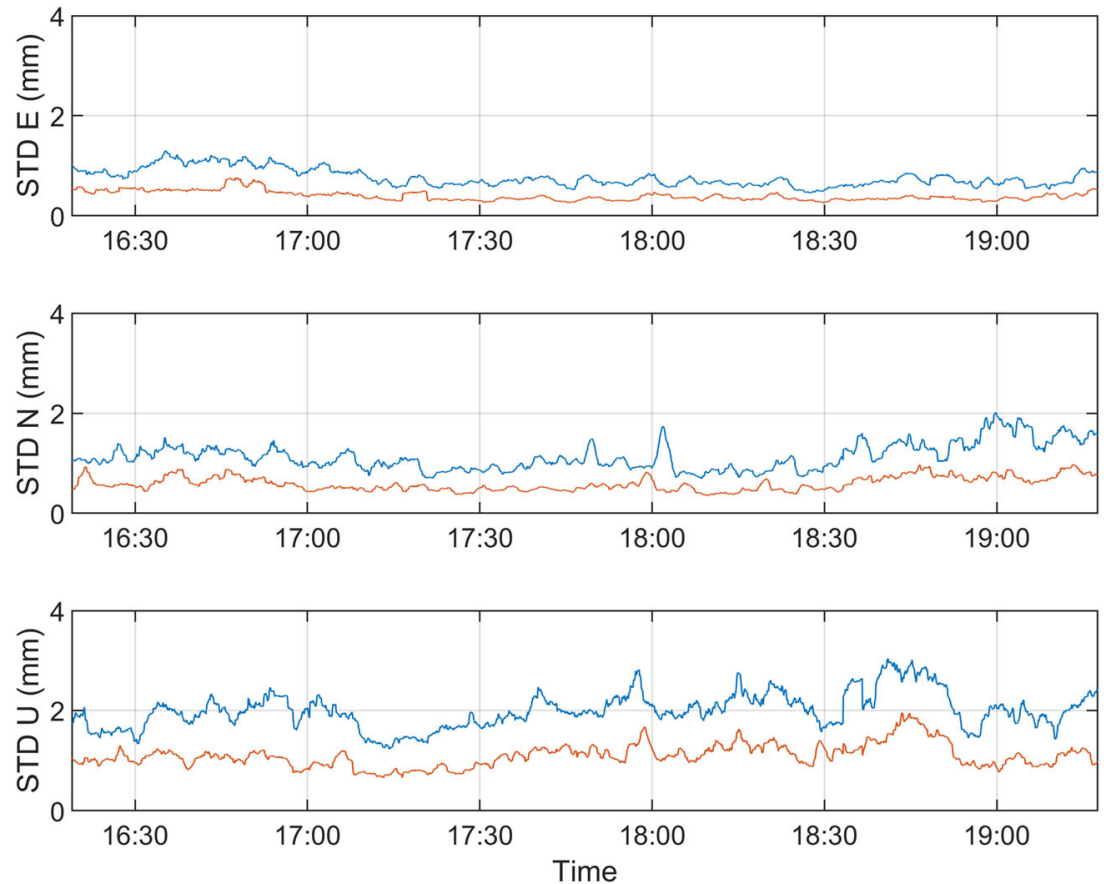
the precision of the GPS time-series would improve through various approaches; (i) use high-end base station, consisting of dual frequency receiver and geodetic antenna, (ii) use dual low-cost GNSS rover-system and application of CME, SDF or both. From the time-series, it is evident the enhancement of the precision of the GPS solution by using the dual low-cost GNSS rover system and applying the CME and SDF methods between the two closely spaced GNSS receivers. The precision of the GPS/GNSS time-series of u-blox receivers with the application of the SDF and/or CME approaches is increased significantly reaching even sub-mm level for the horizontal components and 1-2 $\mathrm{mm}$ level for the vertical component (Table 12). It is even better than the precision which is achieved when geodetic antenna and receiver are used for the GNSS base station. The enhancement of the GPSsolution precision is across the entire frequency band of recording; the level of the coloured and white noise is significantly reduced (Fig. 21).
Fig. 20 The u-blox 2 GPS-only coordinate time series for different analysis approaches: (red) initial GPS-only time series with low-cost GNSS base station, (yellow) GPS-only time series with geodetic receiver and antenna for base station, (blue) CME GPS-only time series, (black) SDF GPS-only time series and (cyan) CME and SDF GPS-only time series. It is evident that the application of the combination of CME and SDF leads to the highest precision of 1-2 mm-level
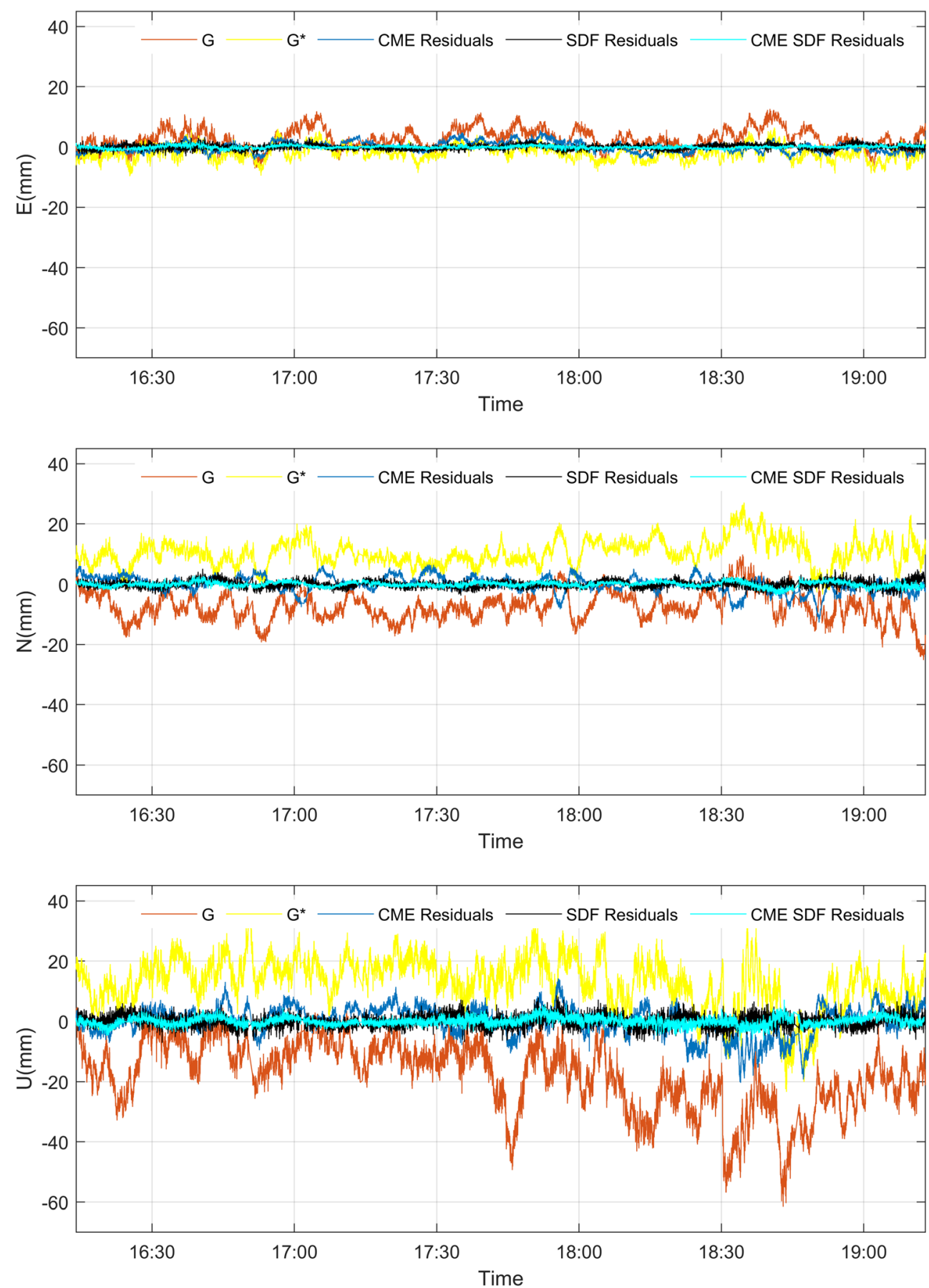
Table 12 The standard deviation (STD) of the GNSS coordinate time series of Day2 for the different approaches: (i) GPS-only solution having low-cost base station, (ii) GPS-only solution having geodetic grade base station, (iii) CME GNSS time series of different satellite constellations (GPS-only, GPS+Galileo and GPS+GLONASS+Galileo), (iv) SDF GPSonly time series, and (v) CME and SDF GPS-only time series. Similar results are for day 1 and day 3

\begin{tabular}{|c|c|c|c|c|c|c|}
\hline \multirow[t]{2}{*}{ STD (mm) } & \multicolumn{3}{|c|}{ u-blox1 } & \multicolumn{3}{|c|}{ u-blox2 } \\
\hline & $\mathrm{E}$ & $\mathrm{N}$ & $\mathrm{U}$ & $\mathrm{E}$ & $\mathrm{N}$ & $\mathrm{U}$ \\
\hline G (u-blox base) & 3.3 & 5.7 & 9.7 & 3.3 & 4.8 & 11.4 \\
\hline G (Leica base) & 2.7 & 5.0 & 8.6 & 2.6 & 4.6 & 8.6 \\
\hline CME G & 1.7 & 2.7 & 4.9 & 1.7 & 2.7 & 4.9 \\
\hline $\mathrm{CME} \mathrm{G}+\mathrm{E}$ & 1.4 & 2.0 & 4.6 & 1.4 & 2.0 & 4.6 \\
\hline $\mathrm{CME} \mathrm{G}+\mathrm{R}+\mathrm{E}$ & 1.2 & 1.7 & 3.9 & 1.2 & 1.5 & 3.5 \\
\hline SDF & 1.0 & 1.7 & 2.9 & 0.8 & 1.2 & 2.0 \\
\hline CME-SDF & 0.5 & 0.9 & 1.5 & 0.5 & 0.9 & 1.5 \\
\hline
\end{tabular}

\section{Conclusions}

The aim of the current study was to evaluate the impact of (i) satellite, (ii) the antenna grade, and (iii) the GNSS base station grade (geodetic antenna-receiver or low-cost receiver and antenna patch), on the precision of the low-cost GNSS receivers and then develop a novel methodology and improve the precision of the low-cost GNSS stations, in order to broaden their application in geodetic deformation monitoring. The approach adopted was based on the theory that two low-cost GNSS stations, with short in-between distance, will be affected by similar error sources, with multipath being the main one which might differ slightly between the two stations. Hence, by modelling the common error between the two low-cost GNSS receivers can lead to the limitation of their noise level. This approach led to the formation of the dual low-cost GNSS rover system, which is combined by two closely spaced (30 cm distance) low-cost GNSS stations and can operate as a single GNSS station. Practically, the second GNSS rover station is used only to model the noise characteristics and limit noise impact on the GNSS coordinate time-series.

The developed method of dual low-cost GNSS rover system improves the performance of low-cost GNSS stations for deformation monitoring applications. One of the main advantages of adopting the developed method is that the existing GNSS monitoring networks can become denser by adding low-cost GNSS rover stations. Furthermore, the developed method and the broad use of low-cost GNSS receivers can make the GNSS monitoring more feasible for structural deformation applications, such as dam monitoring, where until now the high-cost of the permanent geodetic GNSS network limited its application, and other geohazard applications, such as regional ground subsidence, earthquake, landslides, volcanoes (Benoit et al. 2015; Cina and Piras 2015; Janssen et al. 2002; Wilkinson et al. 2017). and meteorological applications (e.g., troposphere estimation (Wilgan and Geiger 2019)).

The current study proved that the developed method of closely spaced low-cost GNSS stations can enhance its precision by examining some of the parameters (antenna, multi-GNSS, SDF, $\mathrm{CME}$ ). However, further investigation is required to assess whether the formation of the low-cost GNSS stations can be
Fig. 21 Spectra of the E, N and Up time series of u-blox2 receiver, for different solutions: (i) GPS-only, (ii) CME GPS-only solution, (iii) SDF GPS-only solution, and (iv) CME and SDF GPS-only solution. The application of CME and SDF leads to the spectrum with the lowest colored and white noise level
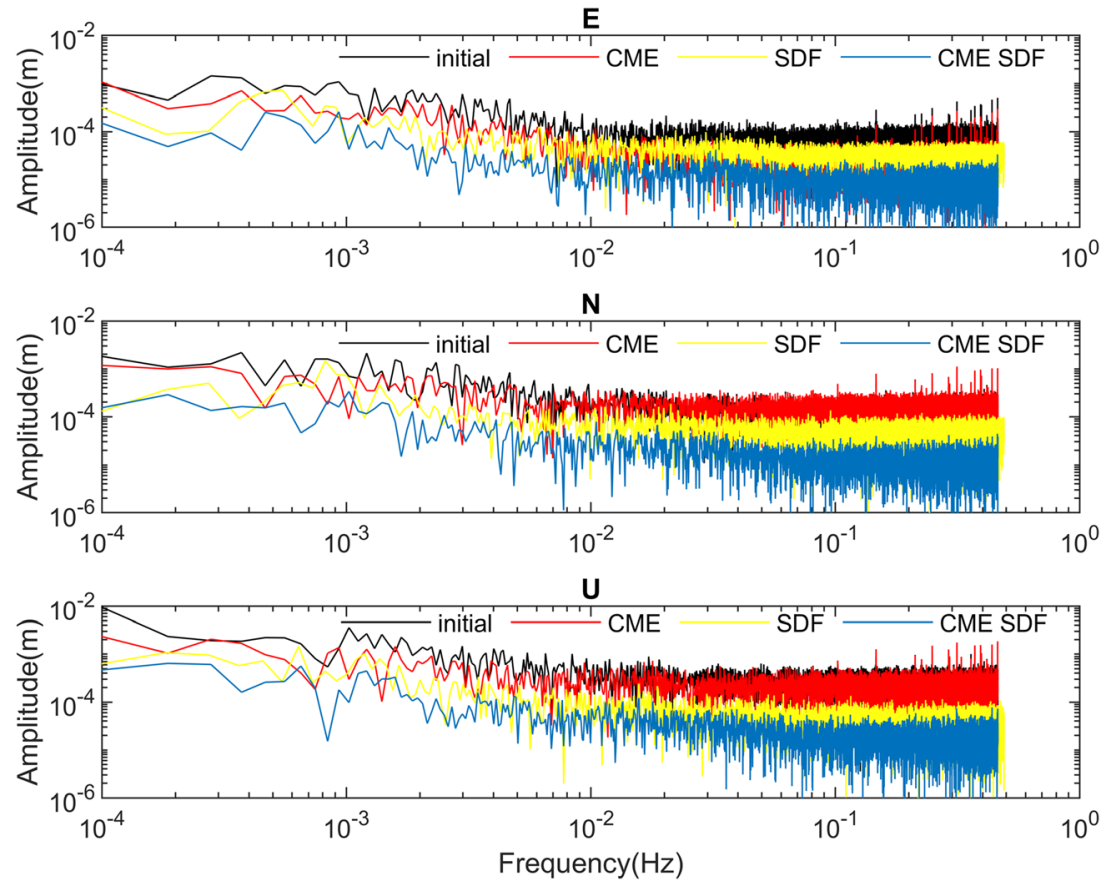
improved or optimised by including more stations. Also, the SDF was only applied for the GPS satellite constellation restricting the application. Potential use of SDF for multiGNSS observations (Galileo, GLONASS, etc.) may enhance even further the precision by using multi-GNSS solution and not only GPS-only solution(Msaewe et al. 2017). Finally, a more advanced modified version of CME, potential application of spatial analysis techniques (Habboub et al. 2020), and the geometric constrain of the short-baseline between the two closely spaced low-cost receivers may lead to more efficient modelling of the common mode error (Zhang et al. 2019).

Supplementary Information The online version contains supplementary material available at https://doi.org/10.1007/s12518-021-00361-8.

Acknowledgments This study has been supported by the Royal Society grants in the framework of the project "Reliability of single frequency GNSS precise positioning for ultra-range bridge deformation monitoring" (RA45UE).

Funding This study has been supported by the Royal Society grants in the framework of the project "Reliability of single frequency GNSS precise positioning for ultra-range bridge deformation monitoring" (RA45UE).

Data availability The data that support the findings of this study are available from the corresponding author, $[\mathrm{Xue}, \mathrm{C}]$, upon reasonable request.

\section{Compliance with ethical standards}

Conflict of interest The authors declare that they have no conflict of interest.

Code availability Any previously unreported custom computer code or algorithm used to generate results that are reported in the paper and central to its main claims are available from the corresponding author, [Xue,C], upon reasonable request.

Open Access This article is licensed under a Creative Commons Attribution 4.0 International License, which permits use, sharing, adaptation, distribution and reproduction in any medium or format, as long as you give appropriate credit to the original author(s) and the source, provide a link to the Creative Commons licence, and indicate if changes were made. The images or other third party material in this article are included in the article's Creative Commons licence, unless indicated otherwise in a credit line to the material. If material is not included in the article's Creative Commons licence and your intended use is not permitted by statutory regulation or exceeds the permitted use, you will need to obtain permission directly from the copyright holder. To view a copy of this licence, visit http://creativecommons.org/licenses/by/4.0/.

\section{References}

Al-Shaery A, Zhang S, Rizos C (2013) An enhanced calibration method of GLONASS inter-channel bias for GNSS RTK. GPS Solutions 17: $165-173$
Amiri-Simkooei A, Tiberius C (2007) Assessing receiver noise using GPS short baseline time series. GPS Solutions 11:21-35

Benoit L, Briole P, Martin O, Thom C, Malet JP, Ulrich P (2015) Monitoring landslide displacements with the Geocube wireless network of low-cost GPS. Eng Geol 195:111-121. https://doi.org/10. 1016/j.enggeo.2015.05.020

Biagi L, Grec FC, Negretti M (2016) Low-Cost GNSS Receivers for Local Monitoring: Experimental Simulation, and Analysis of Displacements. Sensors (Basel) 16. https://doi.org/10.3390/ s16122140

Caldera S, Realini E, Barzaghi R, Reguzzoni M, Sansò F (2016) Experimental Study on Low-Cost Satellite-Based Geodetic Monitoring over Short Baselines. J Surv Eng 142:04015016. https://doi.org/10.1061/(asce)su.1943-5428.0000168

Choi K, Bilich A, Larson KM, Axelrad P (2004) Modified sidereal filtering: Implications for high-rate GPS positioning. Geophys Res Lett 31. https://doi.org/10.1029/2004g1021621

Cina A, Piras M (2015) Performance of low-cost GNSS receiver for landslides monitoring: test and results Geomatics. Nat Hazards Risk 6:497-514

Eissfeller B, Ameres G, Kropp V, Sanroma D (2007) Performance of GPS, GLONASS and Galileo. In: Photogrammetric Week, pp 185-199

Garrido-Carretero MS, de Lacy-Pérez de los Cobos MC, BorqueArancón MJ, Ruiz-Armenteros AM, Moreno-Guerrero R, GilCruz AJ (2019) Low-cost GNSS receiver in RTK positioning under the standard ISO-17123-8: A feasible option in geomatics. Measurement 137:168-178. https://doi.org/10.1016/j. measurement.2019.01.045

Gourevitch S (1996) Innovation: Measuring GPS receiver performance-a new approach gps world 7:56-62

Habboub M, Psimoulis PA, Bingley R, Rothacher M (2020) A Multiple Algorithm Approach to the Analysis of GNSS Coordinate Time Series for Detecting Geohazards and Anomalies. J Geophys Res Solid Earth 125:e2019JB018104. https://doi.org/10.1029/ 2019jb018104

Häberling S, Rothacher M, Zhang Y, Clinton J, Geiger A (2015) Assessment of high-rate GPS using a single-axis shake table. J Geod 89:697-709

Janssen V, Roberts C, Rizos C, Abidin HZ (2002) Low-cost GPS-based volcano deformation monitoring at Mt. Papandayan, Indonesia. J Volcanol Geotherm Res 115:139-151

Jo H, Sim SH, Tatkowski A, Spencer B, Nelson ME (2013) Feasibility of displacement monitoring using low-cost GPS receivers. Struct Control Health Monit 20:1240-1254

Krietemeyer A, ten Veldhuis M-C, van der Marel H, Realini E, van de Giesen N (2018) Potential of Cost-Efficient Single Frequency GNSS Receivers for Water Vapor Monitoring. Remote Sens 10. https://doi.org/10.3390/rs10091493

Leica Geosystems AG (2012) Leica Viva GNSS GS10 receiver Datasheet [online] Heerbrugg, Switzerland

Meng X, Nguyen D, Xie Y, Owen J, Psimoulis P, Ince S, Chen Q, Ye J, Bhatia P (2018) Design and implementation of a new system for large bridge monitoring - GeoSHM. Sensors 18:775

Moschas F, Stiros S (2011) Measurement of the dynamic displacements and of the modal frequencies of a short-span pedestrian bridge using GPS and an accelerometer. Eng Struct 33:10-17. https://doi.org/10. 1016/j.engstruct.2010.09.013

Moschas F, Stiros S (2013) Noise characteristics of high-frequency, short-duration GPS records from analysis of identical, collocated instruments. Measurement 46:1488-1506

Msaewe HA, Hancock CM, Psimoulis PA, Roberts GW, Bonenberg L, de Ligt H (2017) Investigating multi-GNSS performance in the UK and China based on a zero-baseline measurement approach. Measurement 102:186-199. https://doi.org/10.1016/j. measurement.2017.02.004 
Nikolaidis R (2002) Observation of Geodetic and Seismic Deformation with the Global Positioning System

Odolinski R, Teunissen PJ (2017) Low-cost, high-precision, singlefrequency GPS-BDS RTK positioning. GPS Solutions 21:13151330

Peppa I, Psimoulis P, Meng X (2018) Using the signal-to-noise ration of GPS records to detect motion of structures. Struct Control Health Monit 25(2):e2080

Psimoulis PA, Stiros SC (2008) Experimental assessment of the accuracy of GPS and RTS for the determination of the parameters of oscillation of major structures. Comput-Aided Civil Infrastruct Eng 23: 389-403

Psimoulis P, Pytharouli S, Karambalis D, Stiros S (2008) Potential of Global Positioning System (GPS) to measure frequencies of oscillations of engineering structures. J Sound Vib 318:606-623

Ragheb AE, Clarke PJ, Edwards SJ (2007) GPS sidereal filtering: coordinate- and carrier-phase-level strategies. J Geod 81:325-335. https://doi.org/10.1007/s00190-006-0113-1

Raquet CJF (1996) Multiple reference GPS receiver multipath mitigation technique. In: Presented at ION Annual Meeting. Citeseer, $\mathrm{p} 1$

Roberts GW, Tang X, He X (2018) Accuracy analysis of GPS/BDS relative positioning using zero-baseline measurements. J Global Positioning Syst 16:7. https://doi.org/10.1186/s41445-018-0015-6

Schmid R, Steigenberger P, Gendt G, Ge M, Rothacher M (2007) Generation of a consistent absolute phase-center correction model for GPS receiver and satellite antennas. J Geod 81:781-798

Takahashi K, Thu PTA, Irie H, Yamada T (2015) Development of a lowcost small drone-based laser-scanner system for rice monitoring. In: Proceedings of the ACRS. Citeseer

Takasu T, Yasuda A (2008) Evaluation of RTK-GPS performance with low-cost single-frequency GPS receivers. In: Proceedings of international symposium on GPS/GNSS. pp 852-861
Takasu T, Yasuda A (2009) Development of the low-cost RTK-GPS receiver with an open source program package RTKLIB. In: International symposium on GPS/GNSS. International Convention Center Jeju Korea, pp 4-6

Van Sickle J (2001) GPS for Land Surveyors

Wdowinski S, Bock Y, Zhang J, Fang P, Genrich J (1997) Southern California permanent GPS geodetic array: Spatial filtering of daily positions for estimating coseismic and postseismic displacements induced by the 1992 Landers earthquake. J Geophys Res Solid Earth 102:18057-18070

Wilgan K, Geiger A (2019) High-resolution models of tropospheric delays and refractivity based on GNSS and numerical weather prediction data for alpine regions in Switzerland. J Geod 93:819-835

Wilkinson MW et al (2017) Near-field fault slip of the 2016 Vettore M w 6.6 earthquake (Central Italy) measured using low-cost GNSS. Sci Rep 7:4612

Willi D, Rothacher M (2017) GNSS attitude determination with nonsynchronized receivers and short baselines onboard a spacecraft. GPS Solutions 21:1605-1617. https://doi.org/10.1007/s10291017-0639-0

Williams SDP et al (2004) Error analysis of continuous GPS position time series. J Geophys Res Solid Earth 109. https://doi.org/10.1029/ 2003JB002741

Zhang L, Schwieger V (2016) Improving the Quality of Low-Cost GPS Receiver Data for Monitoring Using Spatial Correlations vol 10. https://doi.org/10.1515/jag-2015-0022

Zhang Q, Ma C, Meng X, Xie Y, Psimoulis P, Wu L, Yue Q, Dai X (2019) Galileo Augmenting GPS Single-Frequency Single-Epoch Precise Positioning with Baseline Constrain for Bridge Dynamic Monitoring. Remote Sens 11:438

Zhong P, Ding X, Yuan L, Xu Y, Kwok K, Chen Y (2010) Sidereal filtering based on single differences for mitigating GPS multipath effects on short baselines. J Geod 84:145-158 